\title{
Two-Dimensional Thermofield Bosonization
}

\author{
R. L. P. G. Amaral and L. V. Belvedere \\ Instituto de Física \\ Universidade Federal Fluminense \\ Av. Litorânea S/N, Boa Viagem, Niterói, CEP. 24210-340 \\ Rio de Janeiro - Brasil \\ K. D. Rothe \\ Institut für Theoretische Physik \\ Universität Heidelberg \\ Philosophenweg 16, D-69120 Heidelberg \\ Germany
}

August 14, 2018

\begin{abstract}
The main objective of this paper is to obtain an operator realization for the bosonization of fermions in $1+1$ dimensions, at finite, non-zero temperature $T$. This is achieved in the framework of the real time formalism of Thermofield Dynamics. Formally the results parallel those of the $T=0$ case. The well known two-dimensional Fermion-Boson correspondences at zero temperature are shown to hold also at finite temperature. In order to emphasize the usefulness of the operator realization for handling a large class of two-dimensional quantum field-theoretic problems, we contrast this global approach with the cumbersome calculation of the fermion-current two-point function in the imaginary-time formalism and real time formalisms. The calculations also illustrate the very different ways in which the transmutation from Fermi-Dirac to Bose-Einstein statistics is realized.
\end{abstract}




\section{Introduction}

The bosonization of fermions has proven in the past to be a very useful technique for solving quantum field theoretic models in $1+1$ dimensions [1]. Recently this Fermion-Boson mapping has been discussed for the case of a finite Temperature, using the imaginary time formalism [2], and the equivalence of the massive Thirring model and the sine-Gordon theory was shown also to hold at finite temperature. The main purpose of the present paper is to fill a gap in the literature, by considering the operator formulation of two-dimensional bosonization at finite temperature. To this end we shall use a real time formalism of Thermofield Dynamics [3, 4, 5, 6]. This will enable us to give a unified treatment of whole class of two-dimensional models at finite temperature.

The paper is organized as follows: We begin in section 2 by computing the two-point function of the current of free fermions within the imaginary time and real time formalisms. In this computation we show how the transmutation from Fermi-Dirac statistics to Bose-Einstein statistics is realized in quite different ways. These calculations also serve to emphasize later the simplifying aspects of the operator thermofield bosonization.

In section 3 we consider the two-dimensional free massless scalar thermofield. The corresponding two-point function exhibits two infrared singularities, one similar to the zero temperature case and a new temperature dependent one, implying an indefinite-metric Hilbert space on which the massless scalar thermofield acts. We then show that one can nevertheless define positive definite Wick ordered exponentials of a massless scalar thermofield, provided we associate with them a conserved charge (superselection rule).

In section 4 we consider the two-dimensional Fermi thermofield and compute the corresponding two-point function. In the zero temperature limit we recover the known results.

In section 5 we then use the Wick-ordered exponentials of the free massless scalar thermofield as building blocks for the operator thermofield bosonization of the free massless Fermi field and show that the Fermi thermofield satisfies the correct statistics. We also compute the fermion thermofield current by a point-splitting limit and verify that the current satisfies the usual bosonization correspondence. As a matter of fact, the well known Fermion-Boson correspondences at zero temperature

$$
\begin{gathered}
\bar{\psi} i \gamma^{\mu} \partial_{\mu} \psi \rightarrow \frac{1}{2}: \partial_{\mu} \phi \partial^{\mu} \phi:, \\
\bar{\psi} \gamma^{\mu} \psi \rightarrow-\frac{1}{\sqrt{\pi}} \epsilon^{\mu \nu} \partial_{\nu} \phi, \\
M \bar{\psi} \psi \rightarrow-\frac{\mu M}{\pi}: \cos 2 \sqrt{\pi} \phi:,
\end{gathered}
$$

are shown to hold also at finite temperature.

In section 6 we illustrate the use of thermofield bosonization by solving the massless Thirring model at finite temperature and conclude with comments and outlook in section 7 . We leave to the appendix the computation of the two-point function of the free massive scalar thermofield, and show that the massless scalar thermofield two-point function is recovered in the zero mass limit. 


\section{The Transmutation of Fermi-Dirac to Bose-Einstein Statis- tics}

In this section we illustrate how the transmutation from Fermi-Dirac statistics to Bose-Einstein statistics is realized in the imaginary and real time formalisms in quite different ways. These calculations also serve to emphasize later the simplifying aspects of the operator thermofield bosonization.

\subsection{Current 2-point function in the imaginary time formalism}

In the following it is convenient to work in light-cone coordinates $x_{ \pm}=x_{1} \pm i x_{2}$. Defining correspondingly $j_{ \pm}=j_{1} \pm i j_{2}$, we have for the euclidean two-point current correlator at zero temperature

$$
\begin{aligned}
<j_{+}(x) j_{+}(y)> & =-\operatorname{tr}\left(\gamma_{+} i S_{E}(x-y) \gamma_{+} i S_{E}(y-x)\right) \\
& =\int \frac{d^{2} k}{(2 \pi)^{2}} \Pi_{++}^{(E)}(k) e^{i k \cdot(x-y)}
\end{aligned}
$$

where the overall minus sign arises from the Fermi statistics, and where, after performing the trace using $\not=\frac{1}{2}\left(\gamma_{+} q_{-}+\gamma_{-} q_{+}\right)$and $\gamma_{+}^{2}=\gamma_{-}^{2}=0$,

$$
\Pi_{++}^{(E)}(k)=4 \int \frac{d^{2} p}{(2 \pi)^{2}}\left[(p-k)_{1}+i(p-k)_{2}\right]\left[p_{1}+i p_{2}\right] D_{E}(p-k) D_{E}(p)
$$

with

$$
D_{E}(q)=\frac{1}{q^{2}}=\frac{1}{\left(q_{1}+i q_{2}\right)\left(q_{1}-i q_{2}\right)}
$$

At finite temperature (2.4) is replaced by

$$
<j_{+}(x) j_{+}(y)>_{\beta}=\sum_{\ell} \frac{1}{\beta} \int \frac{d k_{1}}{2 \pi} e^{i k_{1}\left(x_{1}-y_{1}\right)} e^{i \omega_{\ell}\left(x_{2}-y_{2}\right)} \tilde{\Pi}_{++}^{(E)}\left(k_{1}, \omega_{\ell}\right)
$$

where $\omega_{\ell}$ are the Matsubara frequencies $\omega_{\ell}=\frac{2 \pi \ell}{\beta}$, with $\beta=1 / T$ the inverse of the absolute temperature, and

$$
\tilde{\Pi}_{++}^{(E)}\left(k_{1}, \omega_{\ell}\right)=4 \sum_{m} \frac{1}{\beta} \int \frac{d p_{1}}{2 \pi} \frac{1}{\left[p_{1}-i\left(m+\frac{1}{2}\right) \frac{2 \pi}{\beta}\right]\left[p_{1}-k_{1}-i\left(m-\ell+\frac{1}{2}\right) \frac{2 \pi}{\beta}\right]} .
$$

The evaluation of this expression requires regularization. Indeed, as is well known, formally the result depends on the order in which the $p_{1}$ integration and summation over $m$ is being done.

Perform first the summation over $m$

It is convenient to rewrite this expression as

$$
\tilde{\Pi}_{++}^{(E)}\left(k_{1}, \omega_{\ell}\right)=4\left(\frac{i \beta}{2 \pi}\right)^{2} \sum_{m} \frac{1}{\beta} \int \frac{d p_{1}}{2 \pi} \frac{1}{\left[m+\frac{1}{2}+i \frac{\beta}{2 \pi} p_{1}\right]\left[m+\frac{1}{2}+i \frac{\beta}{2 \pi}\left(p_{1}-k_{1}+i \frac{2 \pi}{\beta} \ell\right)\right]}
$$


Making use of the familiar formula 6

$$
\sum_{m} \frac{1}{\left[\left(m+\frac{1}{2}\right)+i x\right]} \frac{1}{\left[\left(m+\frac{1}{2}\right)+i y\right]}=\frac{\pi}{x-y}(\tanh \pi x-\tanh \pi y)
$$

we obtain

$$
\tilde{\Pi}_{++}^{(E)}\left(k_{1}, \omega_{\ell}\right)=-\frac{2}{k_{1}-i \frac{2 \pi \ell}{\beta}} \int \frac{d p_{1}}{2 \pi}\left(\tanh \frac{\beta}{2} p_{1}-\tanh \frac{\beta}{2}\left(p_{1}-k_{1}\right)\right) .
$$

Now

$$
\tanh \frac{\beta}{2} q_{1}=\epsilon\left(q_{1}\right)\left(1-2 N_{F}\left(\left|q_{1}\right|\right)\right)
$$

where $N_{F}\left(\left|q_{1}\right|\right)$ is the Fermi-Dirac distribution

$$
N_{F}\left(\left|q_{1}\right|\right)=\frac{1}{e^{\beta\left|q_{1}\right|}+1} .
$$

Proceeding with the $p_{1}$ integration, one finds

$$
\tilde{\Pi}_{++}^{(E)}\left(k_{1}, \omega_{\ell}\right)=-\frac{2}{\pi} \frac{k_{1}}{k_{1}-i \frac{2 \pi \ell}{\beta}}
$$

A similar result is obtained in this way in the $T=0$ case 7 .

Perform first the integral over $p_{1}$

Performing first the integral in $p_{1}$, one obtains

$$
\begin{aligned}
& \int \frac{d p_{1}}{2 \pi} \frac{1}{\left[p_{1}-i\left(m+\frac{1}{2}\right) \frac{2 \pi}{\beta}\right]\left[p_{1}-k_{1}-i\left(m-\ell+\frac{1}{2}\right) \frac{2 \pi}{\beta}\right]}= \\
& =-i[\theta(2 m+1) \theta(-2 m+2 \ell-1)-\theta(-2 m-1) \theta(2 m-2 \ell+1)] \frac{1}{k_{1}-i \frac{2 \pi \ell}{\beta}}
\end{aligned}
$$

Summing now over $m$ one finds,

$$
\begin{aligned}
\tilde{\Pi}_{++}^{(E)}\left(k_{1}, \omega_{\ell}\right) & =\frac{-4 i}{\beta}(\theta(\ell)+\theta(-\ell-1)) \frac{\ell}{k_{1}-i \frac{2 \pi \ell}{\beta}} \\
& =-\frac{2}{\pi} \frac{i \frac{2 \pi \ell}{\beta}}{k_{1}-i \frac{2 \pi \ell}{\beta}}
\end{aligned}
$$

This is again the analogue of the corresponding $T=0$ result [7].

Averaging now the results we obtain 


$$
\tilde{\Pi}_{++}\left(k_{1}, \omega_{\ell}\right)=-\frac{1}{\pi} \frac{k_{1}+i \frac{2 \pi \ell}{\beta}}{k_{1}-i \frac{2 \pi \ell}{\beta}}
$$

As the final step it remains to compute

$$
<j_{+}(x) j_{+}(y)>_{\beta}=-\frac{1}{\pi} \sum_{\ell} \frac{1}{\beta} \int \frac{d k_{1}}{2 \pi} e^{i k_{1}\left(x_{1}-y_{1}\right)} e^{i \frac{2 \pi \ell}{\beta}\left(x_{2}-y_{2}\right)} \frac{k_{1}+i \frac{2 \pi \ell}{\beta}}{k_{1}-i \frac{2 \pi \ell}{\beta}} .
$$

We may write this as

$$
<j_{+}(x) j_{+}(y)>_{\beta}=-\frac{1}{\pi} \partial_{+}^{2} D_{E}^{(\beta)}(x-y)
$$

where

$$
D_{E}^{(\beta)}(z)=\sum_{\ell} \frac{1}{\beta} \int \frac{d k_{1}}{2 \pi} \frac{e^{i k_{1} z_{1}} e^{i \frac{2 \pi \ell}{\beta} z_{2}}}{\left(k_{1}^{2}+\frac{2 \pi \ell}{\beta}\right)^{2}}
$$

The result, valid for $\left|z_{2}\right|<\beta$ is well known. Replacing the sum by an integral by using a type of Sommerfeld-Watson transform, one finds,

$$
D_{E}^{(\beta)}(z)=\int \frac{d^{2} k}{(2 \pi)^{2}} e^{i k \cdot z}\left[\frac{1}{k^{2}}-i 2 \pi \delta\left(k^{2}\right) N_{B}\left(\left|k_{1}\right|\right)\right]
$$

where $N_{B}\left(\left|k_{1}\right|\right)$ is now the Bose-Einstein distribution function

$$
N_{B}\left(\left|k_{1}\right|\right)=\frac{1}{e^{\beta\left|k_{1}\right|}-1} .
$$

Continuing to Minkowski space this is just the finite-temperature scalar two point function of the real-time formalism.

$$
D_{E}^{(\beta)}(z) \rightarrow i D_{B}^{(\beta)}(z)=i \int \frac{d^{2} k}{(2 \pi)^{2}} e^{-i k \cdot z}\left[\frac{1}{k^{2}+i \epsilon}+2 \pi i \delta\left(k^{2}\right) N_{B}\left(\left|k_{1}\right|\right)\right]
$$

so that

$$
<T j_{+}(x) j_{+}(y)>_{\beta}=\frac{1}{\pi} \partial_{+}^{2} i D_{B}^{(\beta)}(x-y)
$$

We have thus verified the correspondence (1.2) in the imaginary-time formalism, witnessing the metamorphosis from the Fermi-Dirac distribution describing the intermediate fermionic states to be Bose-Einstein distribution in the equivalent bosonic description! The demonstration for the - - component of the current correlator proceeds in the same way. Since the +- components vanish because $\gamma_{ \pm}^{2}=0$, this proves the full equivalence.

We now show that in a quite different, and surprising way, this metamorphosis is realized in the real-time formalism. 


\subsection{Current 2-point function in the real time formalism}

Using the standard rules of the real-time formalism, we have for the ++ component of the current two-point function,

$$
<T j_{++}(x) j_{++}(y)>_{\beta}=-\operatorname{tr}\left(\gamma_{+} i S_{F}^{(\beta)}(x-y) \gamma_{+} i S_{F}^{(\beta)}(y-x)\right),
$$

where

$$
S_{F}^{(\beta)}(z)=\int d^{2} q q D_{F}^{(\beta)}(q) e^{-i q \cdot z}
$$

with

$$
D_{F}^{(\beta)}(q)=\frac{1}{q^{2}+i \epsilon}+2 \pi i \delta\left(q^{2}\right) N_{F}(|\vec{q}|),
$$

where $N_{F}(|\vec{q}|)$ is the Fermi-Dirac distribution (2.5). Here we use $x^{ \pm}=x^{0} \pm x^{1}$ and correspondingly $\gamma^{ \pm}=\gamma^{0} \pm \gamma^{1}$. Noting again that $\phi=\frac{1}{2}\left(\gamma_{+} q_{-}+\gamma_{-} q_{+}\right)$and $\gamma_{+}^{2}=\gamma_{-}^{2}=0$, we find

$$
<T j_{+}(x) j_{+}(y)>_{\beta}=\int \frac{d^{2} k}{(2 \pi)^{2}} \Pi_{++}(k) e^{-i k \cdot(x-y)}
$$

with

$$
\Pi_{++}(k)=4 \int \frac{d^{2} p}{(2 \pi)^{2}}(p-k)_{+} p_{+} D_{F}^{(\beta)}(p-k) D_{F}^{(\beta)}(p) .
$$

We next compute explicitly the integral in (2.12). Separating into terms independent of the temperature $\left(\Pi_{++}^{(0)}\right)$, linear in the Bose-Einstein distribution $\left(\Pi_{++}^{(1)}\right)$, and quadratic in $N_{F}(|\vec{q}|)\left(\Pi_{++}^{(2)}\right)$, we have

$$
\Pi_{++}(k)=\Pi_{++}^{(0)}(k)+\Pi_{++}^{(1)}(k)+\Pi_{++}^{(2)}(k)
$$

we have

$$
\begin{aligned}
\Pi_{++}^{(0)}(k) & =4 \int \frac{d^{2} p}{(2 \pi)^{2}} \frac{(p-k)_{+} p_{+}}{\left[(p-k)^{2}+i \epsilon\right]\left(p^{2}+i \epsilon\right)} \\
\Pi_{++}^{(1)}(k) & =4 \int \frac{d^{2} p}{(2 \pi)^{2}}\left[\frac{(p-k)_{+} p_{+}}{(p-k)^{2}+i \epsilon}(2 \pi i) N_{F}\left(\left|p_{1}\right|\right) \delta\left(p^{2}\right)\right. \\
& \left.+\frac{(p-k)_{+} p_{+}}{p^{2}+i \epsilon}(2 \pi i) N_{F}\left(\left|p_{1}-k_{1}\right|\right) \delta\left((p-k)^{2}\right)\right] \\
\Pi_{++}^{(2)}(k) & =4 \int \frac{d^{2} p}{(2 \pi)^{2}}(p-k)_{+} q_{+}(2 \pi i) N_{F}\left(\left|p_{1}\right|\right) \delta\left(p^{2}\right)(2 \pi i) N_{F}\left(\left|p_{1}-k_{1}\right|\right) \delta\left((p-k)^{2}\right) .
\end{aligned}
$$

$\Pi_{++}^{(0)}(k)$ can be calculated in a variety of ways. A regularization consistent with the conservation law $\partial_{\mu} j^{\mu}=0$ yields

$$
\Pi_{++}^{(0)}(k)=-\frac{i}{\pi} \frac{k_{+} k_{+}}{k^{2}} .
$$


We now make use of the Plemelj's decomposition

$$
\frac{\alpha}{\alpha\left(x-x_{0}\right)+i \epsilon}=\mathcal{P}\left(\frac{1}{x-x_{0}}\right)-i \pi \epsilon(\alpha) \delta\left(x-x_{0}\right)
$$

in order to rewrite $\Pi_{++}^{(1)}(k)$ and $\Pi_{++}^{(2)}(k)$ in the form

$$
\begin{aligned}
& \Pi_{++}^{(1)}(k)=4 \int \frac{d^{2} p}{(2 \pi)^{2}}\left[\mathcal{P}\left(\frac{1}{(p-k)_{-}}\right)(2 \pi i) N_{F}\left(\left|p_{1}\right|\right) p_{+} \delta\left(p^{2}\right)\right. \\
& \left.+\mathcal{P}\left(\frac{1}{p_{-}}\right)(2 \pi i) N_{F}\left(\left|p_{1}-k_{1}\right|\right)(p-k)_{+} \delta\left((p-k)^{2}\right)\right] \\
& -4 \int \frac{d^{2} p}{(2 \pi)^{2}}(2 \pi i)\left[\epsilon\left(p_{+}\right) \delta\left(p_{-}\right) \epsilon\left(p_{+}-k_{+}\right) \delta\left((p-k)_{-}\right)\left[N_{F}\left(\left|p_{1}\right|\right)+N_{F}\left(\left|p_{1}-k_{1}\right|\right)\right]\right.
\end{aligned}
$$

and

$$
\Pi_{++}^{(2)}(k)=4 \int \frac{d^{2} p}{(2 \pi)^{2}}(2 \pi i) N_{F}\left(\left|p_{1}\right|\right) \epsilon\left(p_{+}\right) \delta\left(p_{-}\right)(2 \pi i) N_{F}\left(\left|p_{1}-k_{1}\right|\right) \epsilon\left(p_{+}-k_{+}\right) \delta\left((p-k)_{-}\right) .
$$

Now,

$$
\begin{aligned}
\mathcal{P}\left(\frac{1}{(p-k)_{-}}\right)(2 \pi i) N_{F}\left(\left|p_{1}\right|\right) p_{+} \delta\left(p^{2}\right) & =-\frac{1}{k_{-}} N_{F}\left(\frac{\left|p_{+}\right|}{2}\right) \epsilon\left(p_{+}\right) \delta\left(p_{-}\right) \\
\mathcal{P}\left(\frac{1}{p_{-}}\right)(2 \pi i) N_{F}\left(\left|p_{1}-k_{1}\right|\right)(p-k)_{+} \delta\left((p-k)^{2}\right) & =\frac{1}{k_{-}} N_{F}\left(\frac{\left|(p-k)_{+}\right|}{2}\right) \epsilon\left(p_{+}-k_{+}\right) \delta\left((p-k)_{-}\right) .
\end{aligned}
$$

Since the left hand side of the above expressions are odd functions of $p_{+}$and $p_{+}-k_{+}$, respectively, they do not contribute to the integral above. In terms of light-cone coordinates we have $d^{2} p=$ $\frac{1}{2} d p_{+} d p_{-}$. Performing the $p_{-}$integration we are left with

$$
\begin{aligned}
\Pi_{++}(k)= & -\frac{i}{\pi} \frac{k_{+} k_{+}}{k^{2}}-4(\pi i)^{2} \delta\left(k_{-}\right) \int \frac{d p_{+}}{(2 \pi)^{2}} \epsilon\left(p_{+}-k_{+}\right) \epsilon\left(p_{+}\right) \\
& {\left[N_{F}\left(\frac{\left|p_{+}\right|}{2}\right)+N_{F}\left(\frac{\left|\left(p_{+}-k_{+}\right)\right|}{2}\right)-2 N_{F}\left(\frac{\left|p_{+}\right|}{2}\right) N_{F}\left(\frac{\left|p_{+}-k_{+}\right|}{2}\right)\right] }
\end{aligned}
$$

We now make the change of variable $\frac{p_{+}}{2}=q+\frac{k}{2}$ with $k=k_{+} / 2$. Let us suppose for the moment that $k_{+}$is positive. We may then split the integral in the following way:

$$
\begin{aligned}
\Pi_{++}(k) & =-\frac{i}{\pi} \frac{k_{+} k_{+}}{k^{2}} \\
& +2 \delta\left(k_{-}\right) \int_{-\infty}^{-\frac{k}{2}} d q\left[N_{F}\left(-q-\frac{k}{2}\right)+N_{F}\left(-q+\frac{k}{2}\right)-2 N_{F}\left(-q-\frac{k}{2}\right) N_{F}\left(-q+\frac{k}{2}\right)\right] \\
& +2 \delta\left(k_{-}\right) \int_{\frac{k}{2}}^{\infty} d q\left[N_{F}\left(q+\frac{k}{2}\right)+N_{F}\left(q-\frac{k}{2}\right)-2 N_{F}\left(q+\frac{k}{2}\right) N_{F}\left(q-\frac{k}{2}\right)\right] \\
& +2 \delta\left(k_{-}\right) \int_{-\frac{k}{2}}^{\frac{k}{2}} d q\left[N_{F}\left(q+\frac{k}{2}\right)+N_{F}\left(\frac{k}{2}-q\right)-2 N_{F}\left(q+\frac{k}{2}\right) N_{F}\left(\frac{k}{2}-q\right)\right]
\end{aligned}
$$


Making now explicit use of the Fermi-Dirac distribution (2.5), we obtain from here

$$
\begin{aligned}
\Pi_{++}(k) & =-\frac{i}{\pi} \frac{k_{+} k_{+}}{k^{2}} \\
& +4 \delta\left(k_{-}\right)\left(\int_{\frac{k}{2}}^{\infty} d q \frac{\cosh \beta \frac{k}{2}}{\cosh \beta \frac{k}{2}+\cosh \beta q}-\int_{0}^{\frac{k}{2}} d q \frac{\cosh \beta q}{\cosh \beta \frac{k}{2}+\cosh \beta q}\right)
\end{aligned}
$$

which can be rewritten in the form

$$
\Pi_{++}(k)=-\frac{i}{\pi} \frac{k_{+} k_{+}}{k^{2}}+2 \delta\left(k_{-}\right)\left[I(k) \cosh \beta \frac{k}{2}-k\right]
$$

where

$$
\begin{aligned}
I(k) & =\int_{-\infty}^{\infty} d q \frac{1}{\cosh \frac{\beta k}{2}+\cosh \beta q} \\
& =\frac{k}{\sinh \beta \frac{k}{2}}
\end{aligned}
$$

Putting things together we thus have

$$
\Pi_{++}(k)=-\frac{i}{\pi} \frac{k_{+} k_{+}}{k^{2}}+2 k_{+} \delta\left(k_{-}\right) \frac{1}{e^{\beta \frac{k_{+}}{2}}-1}
$$

Our analysis was done for $k_{+} \geq 0$. Since our original expression (2.14) is symmetric under $k \rightarrow-k$, we find that in general

$$
\Pi_{++}(k)=-\frac{i}{\pi} \frac{k_{+} k_{+}}{k^{2}}+2\left|k_{+}\right| \delta\left(k_{-}\right) \frac{1}{e^{\beta \frac{k_{+} \mid}{2}}-1}
$$

Noting that

$$
\left|k_{+}\right| \delta\left(k_{-}\right)=k_{+} k_{+} \delta\left(k^{2}\right)
$$

we finally conclude that

$$
\Pi_{++}(k)=-\frac{i}{\pi} k_{+} k_{+}\left(\frac{1}{k^{2}}+2 \pi i \delta\left(k^{2}\right) N_{B}\left(\left|k_{1}\right|\right)\right)
$$

where $N_{B}\left(\left|k_{1}\right|\right)$ is now the Bose-Einstein distribution (2.7). A corresponding result is found for $\Pi_{--}(k)$, while again $\Pi_{+-}=\Pi_{-+}=0$. We thus conclude that

$$
<T j_{\mu}(x) j_{\nu}(y)>_{\beta}=\frac{1}{\pi} \tilde{\partial}_{\mu} \tilde{\partial}_{\nu} i D_{B}^{(\beta)}(x-y)
$$

in accordance with the correspondence (1.2) for finite temperature. Notice that unlike in the case of the imaginary-time formalism, we have now witnessed the actual conversion of the Fermi-Dirac to the Bose-Einstein distribution through an integration process. 


\section{$3 \quad$ Free Massless Scalar Thermofield}

As the first step we shall consider the massless scalar field since it is the building block in the bosonization of two-dimensional quantum field theory models. The construction of a quantum field theory at finite temperature requires doubling the number of fields degrees of freedom [3. 4, 5, 6. This is achieved by introducing fictitious "tilde" operators corresponding to each of the operators describing the system considered. This fictitious system is an identical copy of the original system under consideration, except for the opposite norm of the corresponding field. This entails a doubling of the Hilbert space.

To begin with, let us introduce the free scalar thermal doublet ${ }^{1}$,

$$
\Phi=\left(\begin{array}{c}
\phi \\
\widetilde{\phi}
\end{array}\right)
$$

corresponding to the total Lagrangian density,

$$
\mathcal{L}_{T}=\mathcal{L}-\widetilde{\mathcal{L}}=\frac{1}{2} \partial_{\mu} \phi \partial^{\mu} \phi-\frac{1}{2} \partial_{\mu} \widetilde{\phi} \partial^{\mu} \widetilde{\phi}
$$

with the corresponding equations of motion $\left(\partial_{ \pm}=\partial_{0} \pm \partial_{1}\right)$,

$$
\partial_{+} \partial_{-} \phi(x)=0, \partial_{+} \partial_{-} \tilde{\phi}(x)=0 .
$$

In view of the equations of motion (3.3), the two-dimensional free massless scalar field can be decomposed in terms of left- and right-movers $\left(x^{ \pm}=x^{0} \pm x^{1}\right)$,

$$
\phi(x)=\phi_{L}\left(x^{+}\right)+\phi_{R}\left(x^{-}\right),
$$

and similarly for $\widetilde{\phi}(x)$. In order to simplify the notation, we shall omit the subscripts, which are taken to be implied by the arguments $x^{ \pm}$.

In the quantized theory, the two-dimensional Boson field at zero temperature is described by the field operator ,

$$
\begin{gathered}
\phi(x)=\int_{-\infty}^{+\infty}(d p)\left(f_{p}(x) a\left(p^{1}\right)+f_{p}^{*}(x) a^{\dagger}\left(p^{1}\right)\right) . \\
f_{p}(x)=e^{-i p^{\mu} x_{\mu}} \\
(d p)=\frac{d p^{1}}{\sqrt{(2 \pi)\left(2\left|p^{1}\right|\right)}} .
\end{gathered}
$$

The tilde conjugation is defined by the property

$$
\widetilde{(c a)}=c^{*} \widetilde{a}
$$

\footnotetext{
${ }^{1}$ The conventions used are:
}

$$
\gamma^{0}=\left(\begin{array}{ll}
0 & 1 \\
1 & 0
\end{array}\right), \gamma^{1}=\left(\begin{array}{cc}
0 & 1 \\
-1 & 0
\end{array}\right), \gamma^{5}=\gamma^{0} \gamma^{1}, \epsilon^{01}=1, g^{00}=1, x^{ \pm}=x^{0} \pm x^{1}, \partial_{ \pm}=\partial_{0} \pm \partial_{1} .
$$

For the free massless scalar field $\phi(x)=\phi\left(x^{-}\right)+\phi\left(x^{+}\right)$, and for the pseudo-scalar field $\varphi(x)=\varphi\left(x^{-}\right)-\varphi\left(x^{+}\right)$. 
such that the tilde conjugated field is given by,

$$
\widetilde{\phi}(x)=\int_{-\infty}^{+\infty}(d p)\left(f_{p}^{*}(x) \widetilde{a}\left(p^{1}\right)+f_{p}(x) \widetilde{a}^{\dagger}\left(p^{1}\right)\right) .
$$

At $T=0$ these fields are independent,

$$
[\phi(x), \widetilde{\phi}(y)]=0
$$

The Fock vacuum state is,

$$
|\widetilde{0}, 0\rangle=|\widetilde{0}\rangle \otimes|0\rangle,
$$

with the property,

$$
a\left(p^{1}\right)|0\rangle=0, \quad \widetilde{a}\left(p^{1}\right)|\widetilde{0}\rangle=0 .
$$

Decomposing the free massless fields into left- and right-components, we obtain

$$
\begin{aligned}
& \phi\left(x^{ \pm}\right)=\int_{0}^{\infty} \frac{d p^{1}}{\sqrt{4 \pi\left|p^{1}\right|}}\left[f_{p}\left(x^{ \pm}\right)\left(\begin{array}{c}
a\left(-p^{1}\right) \\
a\left(p^{1}\right)
\end{array}\right)+f_{p}^{*}\left(x^{ \pm}\right)\left(\begin{array}{c}
a^{\dagger}\left(-p^{1}\right) \\
a^{\dagger}\left(p^{1}\right)
\end{array}\right)\right], \\
& \widetilde{\phi}\left(x^{ \pm}\right)=\int_{0}^{\infty} \frac{d p^{1}}{\sqrt{4 \pi\left|p^{1}\right|}}\left[f_{p}^{*}\left(x^{ \pm}\right)\left(\begin{array}{c}
\widetilde{a}\left(-p^{1}\right) \\
\widetilde{a}\left(p^{1}\right)
\end{array}\right)+f_{p}\left(x^{ \pm}\right)\left(\begin{array}{c}
\widetilde{a}^{\dagger}\left(-p^{1}\right) \\
\widetilde{a}^{\dagger}\left(p^{1}\right)
\end{array}\right)\right],
\end{aligned}
$$

where,

$$
f_{p}\left(x^{ \pm}\right)=e^{-i p x^{ \pm}}
$$

and

$$
\left[\phi\left(x^{ \pm}\right), \phi\left(y^{\mp}\right)\right]=\left[\widetilde{\phi}\left(x^{ \pm}\right), \widetilde{\phi}\left(y^{\mp}\right)\right]=0 .
$$

Note that in Eqs. (3.13) and (3.14) the limits of integrations are $[0, \infty)$.

In Thermofield Dynamics the temperature-dependent vacuum is defined by,

$$
|0(\beta)\rangle=U_{B}\left(\theta_{B}\right)|0, \widetilde{0}\rangle,
$$

where the unitary operator $U_{B}\left(\theta_{B}\right)$ is given by,

$$
U_{B}\left(\theta_{B}\right)=e^{-i \mathcal{Q}\left(\theta_{B}\right)}=e^{-\int_{-\infty}^{+\infty} d p^{1}\left(\widetilde{a}\left(p^{1}\right) a\left(p^{1}\right)-a^{\dagger}\left(p^{1}\right) \widetilde{a}^{\dagger}\left(p^{1}\right)\right) \theta_{B}\left(\left|p^{1}\right|, \beta\right)},
$$

and the Bogoliubov parameter $\theta_{B}\left(\left|p^{1}\right|, \beta\right)$ is implicitly defined by

$$
\begin{aligned}
& \sinh \theta_{B}\left(\left|p^{1}\right| ; \beta\right)=\frac{e^{-\beta\left|p^{1}\right| / 2}}{\sqrt{1-e^{-\beta\left|p^{1}\right|}}} \\
& \cosh \theta_{B}\left(\left|p^{1}\right| ; \beta\right)=\frac{1}{\sqrt{1-e^{-\beta\left|p^{1}\right|}}},
\end{aligned}
$$

with the Bose-Einstein statistical weight is given by, 


$$
N_{B}\left(\left|p^{1}\right| ; \beta\right)=\sinh ^{2} \theta_{B}\left(\left|p^{1}\right| ; \beta\right)=\frac{1}{e^{\beta\left|p^{1}\right|}-1} .
$$

The correspondingly transformed annihilation operators are given by,

$$
\begin{aligned}
& a\left(p^{1} ; \beta\right)=U_{B}\left(-\theta_{B}\right) a\left(p^{1}\right) U_{B}\left(\theta_{B}\right)=a\left(p^{1}\right) \cosh \theta_{B}\left(\left|p^{1}\right| ; \beta\right)-\widetilde{a}^{\dagger}\left(p^{1}\right) \sinh \theta_{B}\left(\left|p^{1}\right| ; \beta\right), \\
& \widetilde{a}\left(p^{1} ; \beta\right)=U_{B}\left(-\theta_{B}\right) \widetilde{a}\left(p^{1}\right) U_{B}\left(\theta_{B}\right)=\widetilde{a}\left(p^{1}\right) \cosh \theta_{B}\left(\left|p^{1}\right| ; \beta\right)-a^{\dagger}\left(p^{1}\right) \sinh _{B} \theta\left(\left|p^{1}\right| ; \beta\right) .
\end{aligned}
$$

The vacuum state at finite temperature satisfies

$$
a\left(p^{1} ; \beta\right)|0(\beta)\rangle=0, \quad \widetilde{a}\left(p^{1} ; \beta\right)|0(\beta)\rangle=0 .
$$

Here, and in what follows, $p$ is defined to be $p=\left|p^{1}\right|$.

The thermofield operators, that act on the Fock vacuum $|0, \widetilde{0}\rangle$ are given by,

$$
\begin{aligned}
& \phi\left(x^{ \pm} ; \beta\right)=\phi^{(+)}\left(x^{ \pm} ; \beta\right)+\phi^{(-)}\left(x^{ \pm} ; \beta\right), \\
& \widetilde{\phi}\left(x^{ \pm} ; \beta\right)=\widetilde{\phi}^{(+)}\left(x^{ \pm} ; \beta\right)+\widetilde{\phi}^{(-)}\left(x^{ \pm} ; \beta\right),
\end{aligned}
$$

with

$$
\begin{gathered}
\phi^{( \pm)}\left(x^{ \pm} ; \beta\right)=\phi_{c}^{( \pm)}\left(x^{ \pm} ; \beta\right)-\widetilde{\phi}_{s}^{( \pm)}\left(x^{ \pm} ; \beta\right), \\
\widetilde{\phi}^{( \pm)}\left(x^{ \pm} ; \beta\right)=\widetilde{\phi}_{c}^{( \pm)}\left(x^{ \pm} ; \beta\right)-\phi_{s}^{( \pm)}\left(x^{ \pm} ; \beta\right), \\
\phi_{c}^{( \pm)}\left(x^{ \pm} ; \beta\right)=\int_{0}^{\infty}(d p)\left(\begin{array}{c}
f_{p}\left(x^{ \pm}\right) a(\mp p) \\
f_{p}^{*}\left(x^{ \pm}\right) a^{\dagger}(\mp p)
\end{array}\right) \cosh \theta_{B}(p ; \beta), \\
\phi_{s}^{( \pm)}\left(x^{ \pm} ; \beta\right)=\int_{0}^{\infty}(d p)\left(\begin{array}{c}
f_{p}\left(x^{ \pm}\right) a(\mp p) \\
f_{p}^{*}\left(x^{ \pm}\right) a^{\dagger}(\mp p)
\end{array}\right) \sinh \theta_{B}(p ; \beta), \\
\widetilde{\phi}_{s}^{( \pm)}\left(x^{ \pm} ; \beta\right)=\int_{0}^{\infty}(d p)\left(\begin{array}{c}
f_{p}^{*}\left(x^{ \pm}\right) \widetilde{a}(\mp p) \\
f_{p}\left(x^{ \pm}\right) \widetilde{a} \widetilde{a}^{\dagger}(\mp p)
\end{array}\right) \sinh \theta_{B}(p ; \beta), \\
\widetilde{\phi}_{c}^{(+)}\left(x^{ \pm} ; \beta\right)=\int_{0}^{\infty}(d p)\left(\begin{array}{c}
f_{p}^{*}\left(x^{ \pm}\right) \widetilde{a}(\mp p) \\
f_{p}\left(x^{ \pm}\right) \widetilde{a} \widetilde{a}^{\dagger}(\mp p)
\end{array}\right) \cosh \theta_{B}(p ; \beta) .
\end{gathered}
$$

The commutators of the thermofield are the same as that at zero temperature,

$$
\left[\phi\left(x^{ \pm} ; \beta\right), \phi\left(y^{ \pm} ; \beta\right)\right]=\left[\phi_{c}\left(x^{ \pm} ; \beta\right), \phi_{c}\left(y^{ \pm} ; \beta\right)\right]+\left[\widetilde{\phi}_{s}\left(x^{ \pm} ; \beta\right), \widetilde{\phi}_{s}\left(y^{ \pm} ; \beta\right)\right]=\left[\phi\left(x^{ \pm}\right), \phi\left(y^{ \pm}\right)\right]
$$

so that

$$
\left[\phi\left(x^{ \pm} ; \beta\right), \dot{\phi}\left(y^{ \pm} ; \beta\right)\right]=\left[\phi\left(x^{ \pm}\right), \dot{\phi}\left(y^{ \pm}\right)\right]
$$


and

$$
\left[\phi\left(x^{ \pm} ; \beta\right), \tilde{\phi}\left(y^{ \pm} ; \beta\right)\right]=0,
$$

As in the zero temperature case, the massless scalar themofield carries an identically conserved topological current associated with the Gauss' law

$$
J^{\mu}(x ; \beta)=\partial_{\nu} \epsilon^{\mu \nu} \varphi(x ; \beta),
$$

and the field $\varphi(x ; \beta)$ is the dual of $\phi(x ; \beta)$,

$$
\epsilon^{\mu \nu} \partial_{\nu} \varphi(x ; \beta)=\partial^{\mu} \phi(x ; \beta) .
$$

The corresponding charge is formally given by

$$
\mathcal{Q}_{\beta}=\int J^{0}(x ; \beta) d x^{1} .
$$

\subsection{Scalar Thermofield Two-point Function}

For free fields the $n$-point functions are determined by the two-point function. The diagonal contribution to the Schwinger two-point function is given by,

$$
\begin{aligned}
\left\langle 0(\beta)\left|\phi\left(x^{ \pm}\right) \phi\left(y^{ \pm}\right)\right| 0(\beta)\right\rangle & =\left\langle 0, \widetilde{0}\left|\phi\left(x^{ \pm} ; \beta\right) \phi\left(y^{ \pm} ; \beta\right)\right| \widetilde{0}, 0\right\rangle \\
& =\left[\phi_{c}^{(+)}\left(x^{ \pm} ; \beta\right), \phi_{c}^{(-)}\left(y^{ \pm} ; \beta\right)\right]+\left[\widetilde{\phi}_{s}^{(+)}\left(x^{ \pm} ; \beta\right), \widetilde{\phi}_{s}^{(-)}\left(y^{ \pm} ; \beta\right)\right] \\
& =D_{c}^{(+)}\left(x^{ \pm}-y^{ \pm} ; \beta\right)+\widetilde{D}_{s}^{(+)}\left(x^{ \pm}-y^{ \pm} ; \beta\right)
\end{aligned}
$$

where in our simplified notation,

$$
\begin{aligned}
& D_{c}^{(+)}\left(x^{ \pm}-y^{ \pm} ; \beta\right)=\int_{0}^{\infty} \frac{d p}{(2 \pi)(2 p)} f_{p}\left(x^{ \pm}\right) f_{p}^{*}\left(y^{ \pm}\right) \cosh ^{2} \theta_{B}(p ; \beta), \\
& \widetilde{D}_{s}^{(+)}\left(x^{ \pm}-y^{ \pm} ; \beta\right)=\int_{0}^{\infty} \frac{d p}{(2 \pi)(2 p)} f_{p}^{*}\left(x^{ \pm}\right) f_{p}\left(y^{ \pm}\right) \sinh ^{2} \theta_{B}(p ; \beta) .
\end{aligned}
$$

We get,

$$
\left\langle 0, \widetilde{0}\left|\phi\left(x^{ \pm} ; \beta\right) \phi\left(y^{ \pm} ; \beta\right)\right| \widetilde{0}, 0\right\rangle=D_{o}^{(+)}\left(x^{ \pm}-y^{ \pm}\right)+\frac{1}{2 \pi} \int_{0}^{\infty} \frac{d p}{p}\left[\cos p\left(x^{ \pm}-y^{ \pm}\right)\right] N_{B}(p ; \beta),
$$

where the zero temperature two-point function is given by,

$$
D_{o}^{(+)}\left(x^{ \pm}-y^{ \pm}\right)=-\frac{1}{4 \pi} \ln \left[i \mu\left(x^{ \pm}-y^{ \pm}-i \epsilon\right)\right] .
$$


where $\mu$ is an arbitrary infrared (IR) regulator. Besides the usual infrared divergence, the integral in (3.44) introduces an additional divergence. The integral appearing in (3.44) is given by, ${ }^{2}$,

$$
\begin{gathered}
\frac{1}{2 \pi} \int_{0}^{\infty} \frac{d p}{p}\left[\cos p\left(x^{ \pm}-y^{ \pm}\right)\right] N_{B}(p ; \beta) \\
=\frac{1}{4 \pi} \ln \left(x^{ \pm}-y^{ \pm}\right)-\frac{1}{4 \pi} \ln \left\{\sinh \frac{\pi\left(x^{ \pm}-y^{ \pm}\right)}{\beta}\right\}-\frac{1}{4 \pi} \ln \frac{\beta}{\pi}+\frac{1}{2 \pi} \int_{0}^{\infty} \frac{d p}{p\left(e^{\beta p}-1\right)} .
\end{gathered}
$$

The integral appearing in (3.46) is infrared divergent. We shall introduce an infrared cut-off $\mu^{\prime}$ and define

$$
z\left(\beta, \mu^{\prime}\right)=\int_{\mu^{\prime}}^{\infty} \frac{d p}{p\left(e^{\beta p}-1\right)}=\int_{\mu^{\prime}}^{\infty} \frac{d p}{p} N_{B}(p ; \beta),
$$

which corresponds to the mean number of particles having momenta in the range $\left[\mu^{\prime}, \infty\right]$,

$$
z\left(\beta, \mu^{\prime}\right)=\left\langle 0 ; \beta\left|\mathcal{N}_{B}\right| 0(\beta)\right\rangle
$$

where

$$
\mathcal{N}_{B}=\int_{\mu^{\prime}}^{\infty} \frac{d p}{p} a^{\dagger}(p) a(p)
$$

is the operator for the number of particles. For a fixed temperature the asymptotic behavior of the integral (3.47), besides the logarithmic singularity, exhibits also an algebraic singularity,

$$
z\left(\mu^{\prime} \approx 0 ; \beta\right) \rightarrow \frac{1}{\beta \mu^{\prime}}+\frac{1}{2} \ln \left(\beta \mu^{\prime}\right) .
$$

As we shall see, the integral (3.47) plays a crucial role in determining the selection rule for the correlation functions of Wick exponentials of the free massless scalar thermofield.

From (3.44), (3.46) and (3.47) the two-point function for the left- and right-movers seen to be given by ,

$$
\left\langle 0, \widetilde{0}\left|\phi\left(x^{ \pm} ; \beta\right) \phi\left(y^{ \pm} ; \beta\right)\right| \widetilde{0}, 0\right\rangle=-\frac{1}{4 \pi} \ln \left\{i \mu \frac{\beta}{\pi} \sinh \frac{\pi\left(x^{ \pm}-y^{ \pm}-i \epsilon\right)}{\beta}\right\}+\frac{1}{2 \pi} z\left(\beta, \mu^{\prime}\right) .
$$

For the field $\widetilde{\phi}\left(x^{ \pm}\right)$, we obtain

$$
\left\langle 0, \widetilde{0}\left|\widetilde{\phi}\left(x^{ \pm} ; \beta\right) \widetilde{\phi}\left(y^{ \pm} ; \beta\right)\right| \widetilde{0}, 0\right\rangle=-\frac{1}{4 \pi} \ln \left\{i \mu \frac{\beta}{\pi} \sinh \frac{\pi\left(x^{ \pm}-y^{ \pm}+i \epsilon\right)}{\beta}\right\}-\frac{i}{4}+\frac{1}{2 \pi} z\left(\beta, \mu^{\prime}\right) \text {. }
$$

\footnotetext{
${ }^{2}$ Gradshteyn - 4 Edition, pg. 495, $19 m=1$;

$$
\int_{0}^{\infty} \frac{\cos (a x)-\cos (b x)}{e^{\beta x}-1} \frac{d x}{x}=\frac{1}{2} \ln \frac{a \sinh \frac{b \pi}{\beta}}{b \sinh \frac{a \pi}{\beta}} .
$$
}


The limit $\epsilon \rightarrow 0^{+}$was introduced as a prescription to properly define the zero-temperature limit of the above expression as a tempered distribution. For the space-time contribution, the shortdistance limit $x \approx y$ coincides with the $T \rightarrow 0$ limit,

$$
\left\langle 0, \widetilde{0}\left|\phi\left(x^{ \pm} ; \beta\right) \phi\left(y^{ \pm} ; \beta\right)\right| \widetilde{0}, 0\right\rangle \rightarrow-\frac{1}{4 \pi} \ln \left\{i \mu\left(x^{ \pm}-y^{ \pm}-i \epsilon\right)\right\} .
$$

The two-point function for the scalar thermofield $\phi(x ; \beta)=\phi\left(x^{+} ; \beta\right)+\phi\left(x^{-} ; \beta\right)$ is given by,

$$
\begin{aligned}
\langle 0, \widetilde{0}|\phi(x ; \beta) \phi(y ; \beta)| \widetilde{0}, 0\rangle & =\frac{1}{\pi} z\left(\beta, \mu^{\prime}\right) \\
& -\frac{1}{4 \pi} \ln \left\{-\mu^{2}\left(\frac{\beta}{\pi}\right)^{2} \sinh \left[\frac{\pi\left(x^{+}-y^{+}-i \epsilon\right)}{\beta}\right] \sinh \left[\frac{\pi\left(x^{-}-y^{-}-i \epsilon\right)}{\beta}\right]\right\} .
\end{aligned}
$$

In the zero-temperature limit we obtain the two-point function of the free massless scalar field,

$$
\langle 0, \widetilde{0}|\phi(x ; \beta) \phi(y ; \beta)| \widetilde{0}, 0\rangle_{T \rightarrow 0} \rightarrow\langle 0|\phi(x) \phi(y)| 0\rangle_{o}=-\frac{1}{4 \pi} \ln \left\{-\mu^{2}(x-y)^{2}+i \epsilon\left(x_{0}-y_{0}\right)\right\}
$$

The fact that the short-distance behavior of the thermal two-point function is the same as that for zero temperature is a crucial factor for the success of the thermofield bosonization scheme. The appearance of the additive temperature dependent arbitrary constant in (3.51), $z$, is of no immediate significance since the correlators of the massless scalar field in two dimensions does not satisfy positivity. As we shall show in the following section the dependency on this constant of the Wick ordered exponentials will disappear upon imposing suitable superselection rules.

The off-diagonal contribution for the two-point function is given by ${ }^{3}$,

$$
\begin{gathered}
\left\langle 0, \widetilde{0}\left|\phi\left(x^{ \pm} ; \beta\right) \widetilde{\phi}\left(y^{ \pm} ; \beta\right)\right| \widetilde{0}, 0\right\rangle=-\frac{1}{2 \pi} \int_{0}^{\infty} \cos p\left(x^{ \pm}-y^{ \pm}\right) e^{\frac{\beta p}{2}} N_{B}(\theta, p) \frac{d p}{p}= \\
-\frac{1}{4 \pi} \int_{0}^{\infty} \frac{\cos p\left(x^{ \pm}-y^{ \pm}\right)}{\sinh \frac{\beta}{2} p} \frac{d p}{p} \\
=\frac{1}{4 \pi} \ln \cosh \frac{\pi\left(x^{ \pm}-y^{ \pm}\right)}{\beta}-\frac{1}{4 \pi} f(\beta, \kappa),
\end{gathered}
$$

where $f(\beta, \kappa)$ is the integral,

$$
f(\beta, \kappa)=\int_{\kappa}^{\infty} \frac{1}{\sinh \frac{\beta}{2} p} \frac{d p}{p}=\int_{\kappa}^{\infty} \frac{d p}{p} N_{B}(p ; \beta) e^{\frac{\beta p}{2}} .
$$

where $\kappa$ is a new infrared cutoff. This integral also play an important role in the selection rule of the off-diagonal correlation functions of thermal Wick exponentials. In the limit $T \rightarrow 0$ or $x \approx y$, we get for the space-time dependent contribution,

$$
\left\langle 0, \widetilde{0}\left|\phi\left(x^{ \pm} ; \beta\right) \widetilde{\phi}\left(y^{ \pm} ; \beta\right)\right| \widetilde{0}, 0\right\rangle_{T \rightarrow 0} \rightarrow 0
$$

as expected.

\footnotetext{
${ }^{3}$ Gradshtein pag.494, eq. 18 .
} 


\subsection{Wick Ordered Exponential and Selection Rules}

Let us consider the Wick ordered exponential of the free massless fields $\phi\left(x^{ \pm}\right)$and $\widetilde{\phi}\left(x^{ \pm}\right)$,

$$
\begin{gathered}
W(x ; \lambda)=: e^{i \lambda \phi(x)}: \doteq e^{i \lambda \phi^{(-)}(x)} e^{i \lambda \phi^{(+)}(x)}, \\
\widetilde{W}(x ; \lambda)=: e^{-i \lambda \widetilde{\phi}(x)}: \doteq e^{-i \lambda \widetilde{\phi}^{(-)}(x)} e^{-i \lambda \widetilde{\phi}^{(+)}(x)} .
\end{gathered}
$$

The Wick exponential has to be understood as a formal series of Wick-ordered powers of the field at the exponent such that,

$$
\langle 0|W(x ; \lambda)| 0\rangle=\langle\widetilde{0}|\widetilde{W}(x ; \lambda)| \widetilde{0}\rangle=1 .
$$

The unitary operator $U_{B}\left(\theta_{B}\right)$ acts on the creation and annihilation components as

$$
\begin{aligned}
& U_{B}\left(\theta_{B}\right) \phi^{( \pm)}(x) U_{B}^{-1}\left(\theta_{B}\right)=\phi_{c}^{( \pm)}(x ; \beta)-\widetilde{\phi}_{s}^{(\mp)}(x ; \beta), \\
& U_{B}\left(\theta_{B}\right) \widetilde{\phi}^{(+)}(x) U_{B}^{-1}\left(\theta_{B}\right)=\widetilde{\phi}_{c}^{( \pm)}(x ; \beta)-\phi_{s}^{(\mp)}(x ; \beta) .
\end{aligned}
$$

The thermofield Wick exponentials are then given by,

$$
\begin{aligned}
W(x ; \beta, \lambda)= & U_{B}\left(-\theta_{B}\right) W(x ; \lambda) U_{B}\left(\theta_{B}\right)=\left(e^{i \lambda \phi_{c}^{(-)}(x ; \beta)-i \lambda \widetilde{\phi}_{s}^{(+)}(x ; \beta)}\right)\left(e^{i \lambda \phi_{c}^{(+)}(x ; \beta)-i \lambda \widetilde{\phi}_{s}^{(-)}(x ; \beta)}\right) \\
& =\mathcal{Z}\left(\beta, \mu^{\prime}, \lambda^{2}\right)\left(e^{i \lambda \phi_{c}^{(-)}(x ; \beta)-i \lambda \widetilde{\phi}_{s}^{(-)}(x ; \beta)}\right)\left(e^{i \lambda \phi_{c}^{(+)}(x ; \beta)-i \lambda \widetilde{\phi}_{s}^{(+)}(x ; \beta)}\right) \\
\widetilde{W}(x ; \beta, \lambda)= & U_{B}\left(-\theta_{B}\right) \widetilde{W}(x ; \lambda) U_{B}\left(\theta_{B}\right)=\left(e^{-i \lambda \widetilde{\phi}_{c}^{(-)}(x ; \beta)+i \lambda \phi_{s}^{(+)}(x ; \beta)}\right)\left(e^{-i \lambda \widetilde{\phi}_{c}^{(+)}(x ; \beta)+i \lambda \phi_{s}^{(-)}(x ; \beta)}\right) \\
= & \mathcal{Z}\left(\beta, \mu^{\prime}, \lambda^{2}\right)\left(e^{-i \lambda \widetilde{\phi}_{c}^{(-)}(x ; \beta)+i \lambda \phi_{s}^{(-)}(x ; \beta)}\right)\left(e^{-i \lambda \widetilde{\phi}_{c}^{(+)}(x ; \beta)+i \lambda \phi_{s}^{(+)}(x ; \beta)}\right),
\end{aligned}
$$

where

$$
\mathcal{Z}\left(\beta, \mu^{\prime}, \lambda^{2}\right)=\left.e^{-\lambda^{2}\left[\widetilde{\phi}_{s}^{(+)}(x+\varepsilon ; \beta), \widetilde{\phi}_{s}^{(-)}(x ; \beta)\right]}\right|_{\varepsilon \rightarrow 0}=e^{-\frac{\lambda^{2}}{4 \pi} \int_{\mu^{\prime}}^{\infty} \frac{d k}{k} N_{B}(k ; \beta)},
$$

that is

$$
\mathcal{Z}\left(\beta, \mu^{\prime}, \lambda^{2}\right)=e^{-\frac{\lambda^{2}}{4 \pi} z\left(\beta, \mu^{\prime}\right)},
$$

where $z\left(\beta, \mu^{\prime}\right)$ is the integral appearing in the two point function (3.46). The thermal Wick exponential in then given by,

$$
\begin{gathered}
W(x ; \beta, \lambda)=\mathcal{Z}\left(\beta, \mu^{\prime}, \lambda^{2}\right): e^{i \lambda \phi(x ; \beta)}:, \\
\widetilde{W}(x ; \beta, \lambda)=\mathcal{Z}\left(\beta, \mu^{\prime}, \lambda^{2}\right): e^{-i \lambda \widetilde{\phi}(x ; \beta)}: .
\end{gathered}
$$


Thus, we obtain,

$$
\begin{aligned}
& \langle 0(\beta)|W(x ; \lambda)| 0(\beta)\rangle=\langle 0, \widetilde{0}|W(x ; \beta, \lambda)| \widetilde{0}, 0\rangle=\mathcal{Z}\left(\beta, \mu^{\prime}, \lambda^{2}\right), \\
& \langle 0(\beta)|\widetilde{W}(x ; \lambda)| 0(\beta)\rangle=\langle 0, \widetilde{0}|\widetilde{W}(x ; \beta, \lambda)| \widetilde{0}, 0\rangle=\mathcal{Z}\left(\beta, \mu^{\prime}, \lambda^{2}\right) .
\end{aligned}
$$

As we shall see, the factor $\mathcal{Z}\left(\beta, \mu^{\prime}, \lambda^{2}\right)$ plays a role of a wave function renormalization for the bosonized Fermi thermofield. The exponential is IR dependent on the parameters $\mu$ and $\mu^{\prime}$, such that

$$
N_{\mu, \mu^{\prime}}\left[e^{i \lambda \phi(x ; \beta)}\right]=\left(\frac{\mu^{2}}{\nu^{2}}\right)^{\frac{\lambda^{2}}{4 \pi}} \frac{\mathrm{Z}\left(\beta ; \mu^{\prime}\right)}{\mathrm{Z}\left(\beta ; \nu^{\prime}\right)} N_{\nu, \nu^{\prime}}\left[e^{i \lambda \phi(x ; \beta)}\right],
$$

where $N_{\mu, \mu^{\prime}}\left(N_{\nu, \nu^{\prime}}\right)$ stands for "normal ordering" with respect to the masses $\mu, \mu^{\prime}\left(\nu, \nu^{\prime}\right)$.

In order to display the role played by the cut-off dependent term $\mathcal{Z}\left(\beta, \mu^{\prime}, \lambda^{2}\right)$, let us consider the correlation functions at finite temperature of the Wick exponential,

$$
W\left(x_{j} ; \lambda_{j}\right)=: e^{i \lambda_{j} \phi\left(x_{j}\right)}:
$$

The diagonal contribution is given by,

$$
\begin{aligned}
& \left\langle 0(\beta)\left|\prod_{j=1}^{n} W\left(x_{j} ; \lambda_{j}\right)\right| 0(\beta)\right\rangle=\left\langle 0, \widetilde{0}\left|\prod_{j=1}^{n} W\left(x_{j} ; \beta, \lambda_{j}\right)\right| \widetilde{0}, 0\right\rangle= \\
& e^{-\frac{1}{4 \pi} z\left(\beta, \mu^{\prime}\right) \sum_{j=1}^{n} \lambda_{j}^{2}}\left\langle 0, \widetilde{0}\left|\prod_{j=1}^{n}: e^{i \lambda_{j} \phi_{\beta}\left(x_{j}\right)}:\right| \widetilde{0}, 0\right\rangle= \\
& e^{-\frac{1}{4 \pi} z\left(\beta, \mu^{\prime}\right) \sum_{j=1}^{n} \lambda_{j}^{2}} \prod_{i<j}^{n} e^{-\lambda_{i} \lambda_{j}\left\{\left[\phi_{c}^{(+)}\left(x_{i} ; \beta\right), \phi_{c}^{(-)}\left(x_{j} ; \beta\right)\right]+\left[\widetilde{\phi}_{s}^{(+)}\left(x_{i} ; \beta\right), \widetilde{\phi}_{s}^{(-)}\left(x_{j} ; \beta\right)\right]\right\}}= \\
& e^{-\frac{1}{4 \pi} z\left(\beta, \mu^{\prime}\right) \sum_{j=1}^{n} \lambda_{j}^{2}} \prod_{i<j}^{n} e^{-\lambda_{i} \lambda_{j}\left\{D_{c}^{(+)}\left(x_{i}-x_{j} ; \beta\right)+\widetilde{D}_{s}^{(+)}\left(x_{i}-x_{j} ; \beta\right)\right\}} .
\end{aligned}
$$

Using the expression (3.51) for the thermal two-point function, we obtain,

$$
\begin{aligned}
\left\langle 0(\beta)\left|\prod_{j=1}^{n} W\left(x_{j} ; \lambda_{j}\right)\right| 0(\beta)\right\rangle= & e^{-\frac{1}{4 \pi} z\left(\beta, \mu^{\prime}\right)\left(\sum_{j=1}^{n} \lambda_{j}\right)^{2} \times} \\
& \prod_{i<j}^{n}\left[i \mu \frac{\beta}{\pi} \sinh \frac{\pi\left(x_{i}-x_{j}-i \epsilon\right)}{\beta}\right]^{\frac{\lambda_{i} \lambda_{j}}{4 \pi}} .
\end{aligned}
$$

In order to ensure that the correlation functions of the Wick exponential at finite temperature be independent of $\mu^{\prime}$, we must impose the selection rule,

$$
\sum_{i=1}^{n} \lambda_{i}=0
$$

Thus, we can write, 


$$
\begin{aligned}
& \left\langle 0(\beta)\left|\prod_{j=1}^{n} W\left(x_{j} ; \lambda_{j}\right)\right| 0(\beta)\right\rangle=\delta_{\Sigma \lambda_{i}, 0}\left\langle 0, \widetilde{0}\left|\prod_{j=1}^{n} W\left(x_{j} ; \beta, \lambda_{j}\right)\right| \widetilde{0}, 0\right\rangle= \\
& =\delta_{\Sigma \lambda_{i}, 0}(\mu)^{\sum_{i<j}^{n} \frac{\lambda_{i} \lambda_{j}}{4 \pi}} \prod_{i<j}^{n}\left[\frac{\beta}{\pi} \sinh \frac{\pi\left(x_{i}-x_{j}-i \epsilon\right)}{\beta}\right]^{\frac{\lambda_{i} \lambda_{j}}{4 \pi}} \\
& \equiv \delta_{\Sigma \lambda_{i}, 0} \prod_{j=1}^{n}(\mu)^{-\frac{\lambda_{j}^{2}}{8 \pi}} \prod_{i<j}^{n}\left[\frac{\beta}{\pi} \sinh \frac{\pi\left(x_{i}-x_{j}-i \epsilon\right)}{\beta}\right]^{\frac{\lambda_{i} \lambda_{j}}{4 \pi}}
\end{aligned}
$$

As in the $T=0$ case we may thus define a multiplicatively, infrared renormalized Wick exponential,

$$
W_{R}\left(x_{j} ; \lambda_{j}\right)=(\mu)^{\frac{\lambda_{j}^{2}}{8 \pi}} W\left(x_{j} ; \lambda_{j}\right),
$$

such that,

$$
W_{R}\left(x_{j} ; \beta, \lambda_{j}\right)=(\mu)^{\frac{\lambda_{j}^{2}}{8 \pi}} \mathcal{Z}\left(\beta, \mu^{\prime}, \lambda_{j}^{2}\right): e^{i \lambda_{j} \phi\left(x_{j} ; \beta\right)}: .
$$

It is interesting that one and the same selection rule $\sum_{i} \lambda_{i}=0$ allows us to eliminate the dependence on the infrared regulators $\mu$ and $\mu^{\prime}$. Thus, provided we associate with the Wick exponential a conserved charge $\lambda$, the correlation functions of the thermal Wick exponentials (3.77) are mapped into Wightman functions belonging to a positive metric Hilbert space. In a similar way for the correlation functions of the Wick exponential $\widetilde{W}\left(x_{j} ; \beta, \lambda_{j}\right)$,

$$
\widetilde{W}_{R}\left(x_{j} ; \beta, \tilde{\lambda}_{j}\right)=(\mu)^{\frac{\tilde{\lambda}_{j}^{2}}{8 \pi}} \mathcal{Z}\left(\beta, \mu^{\prime}, \tilde{\lambda}_{j}^{2}\right): e^{-i \tilde{\lambda}_{j} \widetilde{\phi}\left(x_{j} ; \beta\right)}: .
$$

Using Eq. (3.51), the correlation functions of the Wick exponential $\widetilde{W}(x ; \beta, \lambda)$ can be obtained from (3.75) by making $i \epsilon \rightarrow-i \epsilon$ and introducing a phase factor

$$
e^{i \pi \sum_{i<j}^{n} \frac{\lambda_{i} \lambda_{j}}{4 \pi}} .
$$

The off-diagonal correlation functions are given by,

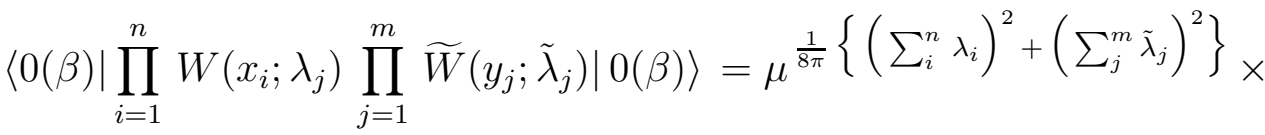

$$
\begin{aligned}
& e^{-\frac{1}{8 \pi} z\left(\beta, \mu^{\prime}\right)\left\{\left(\sum_{i}^{n} \lambda_{i}\right)^{2}+\left(\sum_{j}^{m} \tilde{\lambda}_{j}\right)^{2}\right\}} e^{-\frac{1}{8 \pi} f(\beta, \kappa)\left(\sum_{i}^{n} \lambda_{i}\right)\left(\sum_{j}^{m} \tilde{\lambda}_{j}\right)_{\times}} \\
& \prod_{i<k}^{n}\left[\frac{\beta}{\pi} \sinh \frac{\pi\left(x_{i}-x_{k}\right)}{\beta}\right]^{\frac{\lambda_{i} \lambda_{k}}{4 \pi}} \prod_{j<\ell}^{m}\left[\frac{\beta}{\pi} \sinh \frac{\pi\left(y_{j}-y_{\ell}\right)}{\beta}\right]^{\frac{\tilde{\lambda}_{j} \tilde{\lambda}_{\ell}}{4 \pi}} \times \\
& \prod_{i, j}^{n, m}\left[\cosh \frac{\pi\left(x_{i}-y_{j}\right)}{\beta}\right]^{-\frac{\lambda_{i} \tilde{\lambda}_{j}}{4 \pi}} .
\end{aligned}
$$


Again, the selection rules that makes the off-diagonal correlation function simultaneously independent of $\mu, \mu^{\prime}$ and $\kappa$ are,

$$
\sum_{i=1}^{n} \lambda_{i}=0, \sum_{j=1}^{m} \tilde{\lambda}_{j}=0,
$$

and we get for the IR renormalized Wick exponentials,

$$
\begin{gathered}
\left\langle 0(\beta)\left|\prod_{i=1}^{n} W\left(x_{i} ; \lambda_{i}\right) \prod_{j=1}^{m} \widetilde{W}\left(y_{j} ; \tilde{\lambda}_{j}\right)\right| 0(\beta)\right\rangle=\delta_{\Sigma \lambda_{i}, 0} \delta_{\Sigma \tilde{\lambda}_{j}, 0} \times \\
\prod_{i<k}^{n}\left[i \frac{\beta}{\pi} \sinh \frac{\pi\left(x_{i}-x_{k}\right)}{\beta}\right]^{\frac{\lambda_{i} \lambda_{k}}{4 \pi}} \prod_{j<\ell}^{m}\left[i \frac{\beta}{\pi} \sinh \frac{\pi\left(y_{j}-y_{\ell}\right)}{\beta}\right]^{\frac{\tilde{\lambda}_{j} \tilde{\lambda}_{\ell}}{4 \pi}} \times \\
\prod_{i, j}^{n, m}\left[\cosh \frac{\pi\left(x_{i}-y_{j}\right)}{\beta}\right]^{-\frac{\lambda_{i} \tilde{\lambda}_{j}}{4 \pi}} .
\end{gathered}
$$

\section{Free Massless Fermi Thermofield}

Let us introduce the fermion doublet [4]

$$
\Psi=\left(\begin{array}{c}
\psi \\
i \widetilde{\psi}^{*}
\end{array}\right)
$$

and the corresponding total Lagrangian density,

$$
\mathcal{L}_{T}=i \bar{\Psi} \gamma^{\mu} \partial_{\mu} \Psi=\mathcal{L}-\widetilde{\mathcal{L}}=i \bar{\psi} \gamma_{\mu} \partial_{\mu} \psi-\left(-i \overline{\widetilde{\psi}} \gamma^{\mu} \partial_{\mu} \widetilde{\psi}\right)
$$

The free massless Fermi fields $\psi$ and $\widetilde{\psi}$ are given in terms of right and left spinor components,

$$
\begin{aligned}
& \psi(x)=\left(\begin{array}{l}
\psi\left(x^{+}\right) \\
\psi\left(x^{-}\right)
\end{array}\right), \\
& \widetilde{\psi}(x)=\left(\begin{array}{l}
\widetilde{\psi}\left(x^{+}\right) \\
\widetilde{\psi}\left(x^{-}\right)
\end{array}\right),
\end{aligned}
$$

where

$$
\begin{aligned}
& \psi\left(x^{ \pm}\right)=\int_{0}^{\infty} \frac{d p}{\sqrt{2 \pi}}\left\{f_{p}\left(x^{ \pm}\right) b(\mp p)+f_{p}^{*}\left(x^{ \pm}\right) d^{\dagger}(\mp p)\right\} \\
& \widetilde{\psi}\left(x^{ \pm}\right)=\int_{0}^{\infty} \frac{d p}{\sqrt{2 \pi}}\left\{f_{p}^{*}\left(x^{ \pm}\right) \widetilde{b}(\mp p)+f_{p}\left(x^{ \pm}\right)\right\} \widetilde{d}^{\dagger}(\mp p) .
\end{aligned}
$$

The unitary operator taking one to the thermofields is now given by,

$$
U_{F}\left(\theta_{F}\right)=e^{-\int_{-\infty}^{\infty} d p \theta_{F}\left(\left|p^{1}\right|, \beta\right)\left(\widetilde{b}\left(p^{1}\right) b\left(p^{1}\right)-b^{\dagger}\left(p^{1}\right) \widetilde{b}^{\dagger}\left(p^{1}\right)+\widetilde{d}\left(p^{1}\right) d(p)-d^{\dagger}\left(p^{1}\right) \widetilde{d}^{\dagger}\left(p^{1}\right)\right)},
$$


and the corresponding transformed annihilation operators are

$$
\begin{aligned}
& b\left(p^{1} ; \beta\right)=b\left(p^{1}\right) \cos \theta_{F}(p ; \beta)-\widetilde{b}^{\dagger}\left(p^{1}\right) \sin \theta_{F}(p ; \beta), \\
& \widetilde{b}\left(p^{1}, \beta\right)=\widetilde{b}\left(p^{1}\right) \cos _{F} \theta(p ; \beta)+b^{\dagger}\left(p^{1}\right) \sin \theta_{F}(p ; \beta),
\end{aligned}
$$

with similar expressions for $d\left(p^{1} ; \beta\right)$ and $\widetilde{d}\left(p^{1} ; \beta\right)$, where the Bogoliubov parameter $\theta_{F}(p, \beta)$ is now implicitly defined by,

$$
\begin{aligned}
& \cos \theta_{F}(p ; \beta)=\frac{1}{\sqrt{1+e^{-\beta p}}}, \\
& \sin \theta_{F}(p ; \beta)=\frac{e^{-\beta p / 2}}{\sqrt{1+e^{-\beta p}}},
\end{aligned}
$$

and the Fermi-Dirac statistical weight is given by,

$$
N_{F}(p ; \beta)=\sin ^{2} \theta_{F}(p ; \beta)=\frac{1}{e^{\beta p}+1} .
$$

The fermion thermofields are then

$$
\begin{aligned}
\psi\left(x^{ \pm} ; \beta\right) & =\int_{0}^{\infty} \frac{d p}{\sqrt{2 \pi}}\left\{f_{p}\left(x^{ \pm}\right)\left(b(\mp p) \cos \theta_{F}(p ; \beta)-\widetilde{b}^{\dagger}(\mp p) \sin \theta_{F}(p ; \beta)\right)\right. \\
& \left.+f_{p}^{*}\left(x^{ \pm}\right)\left(d^{\dagger}(\mp p) \cos \theta_{F}(p ; \beta)-\widetilde{d}(\mp p) \sin \theta_{F}(p ; \beta)\right)\right\} .
\end{aligned}
$$

and

$$
\begin{aligned}
\widetilde{\psi}\left(x^{ \pm} ; \beta\right) & =\int_{0}^{\infty} \frac{d p}{\sqrt{2 \pi}}\left\{f_{p}^{*}\left(x^{ \pm}\right)\left(\widetilde{b}(\mp p) \cos \theta_{F}(p ; \beta)+b^{\dagger}(\mp p) \sin \theta_{F}(p ; \beta)\right)\right. \\
& \left.+f_{p}\left(x^{ \pm}\right)\left(\widetilde{d}^{\dagger}(\mp p) \cos \theta_{F}(p ; \beta)+d(\mp p) \sin \theta_{F}(p ; \beta)\right)\right\} .
\end{aligned}
$$

Besides the canonical anticommutation relations we have,

$$
\{\psi(x ; \beta), \widetilde{\psi}(y ; \beta)\}=0,\left\{\psi^{\dagger}(x ; \beta), \widetilde{\psi}(y ; \beta)\right\}=0
$$

\subsection{Fermion Thermofield Two-point Function}

The diagonal contribution for the two-point function of the thermofield fermion is given by,

$$
\left\langle 0, \widetilde{0}\left|\psi\left(x^{ \pm} ; \beta\right) \psi^{\dagger}\left(y^{ \pm} ; \beta\right)\right| 0, \widetilde{0}\right\rangle=\frac{1}{2 i \pi\left(x^{ \pm}-y^{ \pm}\right)}-\frac{1}{i \pi} \int_{0}^{\infty} d p N_{F}(p ; \beta) \sin p\left(x^{ \pm}-y^{ \pm}\right)
$$

The first term in (4.16) is the two-point function at zero temperature,

$$
\left\langle 0\left|\psi^{\dagger}\left(x^{ \pm}\right) \psi\left(y^{ \pm}\right)\right| 0\right\rangle_{0}=\frac{1}{2 i \pi\left(x^{ \pm}-y^{ \pm}\right)} .
$$


The integral appearing in the two-point function (4.16) is evaluated to be ${ }^{4}$

$$
\frac{1}{i \pi} \int_{0}^{\infty} d p N_{F}(p ; \beta) \sin p(x-y)=\frac{1}{2 i \pi(x-y)}-\frac{1}{2 i \beta \sinh \left[\frac{\pi}{\beta}(x-y)\right]} .
$$

We obtain after appropriate $i \epsilon$ prescription,

$$
\begin{aligned}
\left\langle 0, \widetilde{0}\left|\psi\left(x^{ \pm} ; \beta\right) \psi^{\dagger}\left(y^{ \pm} ; \beta\right)\right| 0, \widetilde{0}\right\rangle & =\frac{1}{2 i \beta \sinh \left[\frac{\pi}{\beta}\left(x^{ \pm}-y^{ \pm}-i \epsilon\right)\right]} \\
\left\langle 0, \widetilde{0}\left|\widetilde{\psi}\left(x^{ \pm} ; \beta\right) \widetilde{\psi}^{\dagger}\left(y^{ \pm} ; \beta\right)\right| 0, \widetilde{0}\right\rangle & =\frac{-1}{2 i \beta \sinh \left[\frac{\pi}{\beta}\left(x^{ \pm}-y^{ \pm}+i \epsilon\right)\right]}
\end{aligned}
$$

The diagonal thermofield fermion correlation function satisfies the asymptotic factorization property

$$
\lim _{\lambda \rightarrow \infty}\left\langle 0, \widetilde{0}\left|\psi(x+\lambda ; \beta) \psi^{\dagger}(x ; \beta)\right| 0, \widetilde{0}\right\rangle \rightarrow|\langle 0, \widetilde{0}|\psi(x ; \beta)| 0, \widetilde{0}\rangle|^{2}=0 .
$$

For $T \rightarrow 0$ as well as $x \approx y$ at fixed $T$, we obtain,

$$
\left\langle 0, \widetilde{0}\left|\psi\left(x^{ \pm} ; \beta\right) \psi^{\dagger}\left(y^{ \pm} ; \beta\right)\right| 0, \widetilde{0}\right\rangle \rightarrow \frac{1}{2 i \pi\left(x^{ \pm}-y^{ \pm}-i \epsilon\right)} .
$$

For the off-diagonal contribution we find ${ }^{5}$,

$$
\begin{aligned}
\left\langle 0, \widetilde{0}\left|\widetilde{\psi}\left(x^{ \pm} ; \beta\right) \psi\left(y^{ \pm} ; \beta\right)\right| 0, \widetilde{0}\right\rangle & =-\frac{1}{\pi} \int_{0}^{\infty} \cos p\left(x^{ \pm}-y^{ \pm}\right) N_{F}(\beta, p) e^{-\frac{\beta}{2} p} d p \\
& =-\frac{1}{2 \pi} \int_{0}^{\infty} \frac{\cos p\left(x^{ \pm}-y^{ \pm}\right)}{\cos \frac{\beta}{2} p} d p \\
& =-\frac{1}{2 \beta \cosh \left[\frac{\pi}{\beta}\left(x^{ \pm}-y^{ \pm}\right)\right]}
\end{aligned}
$$

In the limit $T \rightarrow 0$ we get,

$$
\left\langle 0, \widetilde{0}\left|\widetilde{\psi}\left(x^{ \pm} ; \beta\right) \psi\left(y^{ \pm} ; \beta\right)\right| 0, \widetilde{0}\right\rangle_{T \rightarrow 0} \rightarrow 0,
$$

as expected. The off-diagonal two-point function also satisfy the cluster decomposition property. Moreover,

$$
\left\langle 0, \widetilde{0}\left|\widetilde{\psi}\left(x^{ \pm} ; \beta\right) \psi^{\dagger}\left(y^{ \pm} ; \beta\right)\right| 0, \widetilde{0}\right\rangle=0 .
$$

\footnotetext{
${ }^{4}$ Gradshtein - 4 Edition, pag. 481- eq. 3.9911-1,

$$
\int_{0}^{\infty} d x \frac{\sin a x}{e^{\beta x}+1}=\frac{1}{2 a}-\frac{\pi}{2 \beta \sinh \frac{a \pi}{\beta}} .
$$
}

${ }^{5}$ Gradshtein, pag. 503, last integral. 


\section{Thermofield Bosonization of the Free Massless Fermion}

The scale dimension of the thermofield Wick exponential is $d=\frac{\lambda^{2}}{4 \pi}$. In analogy to the $T=0$ case, we seek a representation of the thermal Fermi field in terms of the IR renormalized thermofield Wick exponential of canonical scale dimension $\lambda=2 \sqrt{\pi}$ :

$$
\begin{gathered}
\psi\left(x^{ \pm} ; \beta\right) \doteq W_{R}\left(x^{ \pm} ; \beta\right)=C \mathcal{Z}_{c}\left(\beta, \mu^{\prime}\right): e^{2 i \sqrt{\pi} \phi\left(x^{ \pm} ; \beta\right)}: \\
\widetilde{\psi}\left(x^{ \pm} ; \beta\right) \doteq \widetilde{W}_{R}\left(x^{ \pm} ; \beta\right)=C \mathcal{Z}_{c}\left(\beta, \mu^{\prime}\right): e^{-2 i \sqrt{\pi} \widetilde{\phi}\left(x^{ \pm} ; \beta\right)}:
\end{gathered}
$$

where

$$
\begin{gathered}
W_{R}\left(x^{ \pm} ; \beta\right)=W_{R}\left(x^{ \pm} ; \beta, 2 \sqrt{\pi}\right) \\
\mathcal{Z}_{c}\left(\beta, \mu^{\prime}\right)=\mathcal{Z}\left(\beta, \mu^{\prime}, 4 \pi\right)
\end{gathered}
$$

and

$$
C=\left(\frac{\mu}{2 \pi}\right)^{1 / 2}
$$

Taking into account the super selection rule associated with the Wick exponential of thermofields, one readily verifies that the set of all two-point functions of the Fermi thermofield, obtained in the previous section, are recovered from the bosonized expression (5.1). In particular property (4.25) follows from the superselection rule for the Wick exponential of the Bose ther-

mofield. The minus sign appearing in the two-point function for the thermofield $\widetilde{\psi}(x ; \beta)$ arises from the phase factor (3.79).

\subsection{Statistics}

Let us consider the anticommutation relation between the same spinor components labeled by \pm . From (5.1) we obtain,

$$
\begin{aligned}
\psi\left(x^{ \pm} ; \beta\right) \psi\left(y^{ \pm} ; \beta\right) & =C^{2} \mathcal{Z}_{c}^{2}\left(\beta, \mu^{\prime}\right): e^{2 i \sqrt{\pi} \phi\left(x^{ \pm} ; \beta\right)}:: e^{2 i \sqrt{\pi} \phi\left(y^{ \pm} ; \beta\right)}: \\
& =C^{2} \mathcal{Z}_{c}^{2}\left(\beta, \mu^{\prime}\right): e^{2 i \sqrt{\pi}\left[\phi\left(x^{ \pm} ; \beta\right)+\phi\left(y^{ \pm} ; \beta\right)\right]}: e^{-4 \pi\left\{D_{c}^{(+)}\left(x^{ \pm}-y^{ \pm} ; \beta\right)+\widetilde{D}_{s}^{(+)}\left(x^{ \pm}-y^{ \pm} ; \beta\right)\right\}} \\
& =C^{2} \mathcal{Z}_{c}^{4}\left(\beta, \mu^{\prime}\right): \psi\left(x^{ \pm} ; \beta\right) \psi\left(y^{ \pm} ; \beta\right):\left(i \mu \frac{\beta}{\pi} \sinh \left[\frac{\pi}{\beta}\left(x^{ \pm}-y^{ \pm}-i \epsilon\right)\right]\right) .
\end{aligned}
$$

From the antisymmetry of the $\sinh z$ under $z \rightarrow-z$ it follows that,

$$
\left\{\psi\left(x^{ \pm} ; \beta\right), \psi\left(y^{ \pm} ; \beta\right)\right\}=0
$$

In a similar way, we obtain,

$$
\begin{gathered}
\left\{\psi\left(x^{ \pm} ; \beta\right), \psi^{\dagger}\left(y^{ \pm} ; \beta\right)\right\}=: e^{2 i \sqrt{\pi}\left[\phi\left(x^{ \pm} ; \beta\right)-\phi\left(y^{ \pm} ; \beta\right)\right]}: \times \\
\frac{1}{2 i \beta}\left(\frac{1}{\sinh \left[\frac{\pi}{\beta}\left(x^{ \pm}-y^{ \pm}-i \epsilon\right)\right]}-\frac{1}{\sinh \left[\frac{\pi}{\beta}\left(x^{ \pm}-y^{ \pm}+i \epsilon\right)\right]}\right) .
\end{gathered}
$$

The non zero contributions in Eq. (5.8) arises from the neighborhood of the point $x=y$. This time we get, 


$$
\left\{\psi\left(x^{ \pm} ; \beta\right), \psi^{\dagger}\left(y^{ \pm} ; \beta\right)\right\}=\delta\left(x^{ \pm}-y^{ \pm}\right) .
$$

For the tilde-fields we analogously find,

$$
\begin{gathered}
\left\{\widetilde{\psi}\left(x^{ \pm} ; \beta\right), \widetilde{\psi}\left(y^{ \pm} ; \beta\right)\right\}=0, \\
\left\{\widetilde{\psi}\left(x^{ \pm} ; \beta\right), \widetilde{\psi}^{\dagger}\left(y^{ \pm} ; \beta\right)\right\}=\delta\left(x^{ \pm}-y^{ \pm}\right) .
\end{gathered}
$$

In order to ensure the correct anticommutation relations between $\psi\left(x^{+} ; \beta\right)$ and $\psi\left(x^{-} ; \beta\right)$, between $\widetilde{\psi}\left(x^{+} ; \beta\right)$ and $\widetilde{\psi}\left(x^{-} ; \beta\right)$, as well as the anticummutativity of tilde operators with non-tilde ones, Klein factors [10, 11] must be introduced. To begin with, let us introduce the charges,

$$
\begin{aligned}
& \mathcal{Q}_{\beta}^{ \pm} \doteq \int_{-\infty}^{+\infty} \partial_{0} \phi\left(x^{ \pm} ; \beta\right) d x^{1}, \\
& \widetilde{\mathcal{Q}}_{\beta}^{ \pm} \doteq \int_{-\infty}^{+\infty} \partial_{0} \widetilde{\phi}\left(x^{ \pm} ; \beta\right) d x^{1},
\end{aligned}
$$

and the Klein factors,

$$
\begin{gathered}
K_{\beta}^{ \pm}=e^{i \frac{\sqrt{\pi}}{2} \mathcal{Q}_{\beta}^{ \pm}}, \\
\widetilde{K}_{\beta}^{ \pm}=e^{-i \frac{\sqrt{\pi}}{2} \widetilde{\mathcal{Q}}_{\beta}^{ \pm}} .
\end{gathered}
$$

Since

$$
\mathcal{Q}_{\beta}^{ \pm}=U_{B}(-\theta) \mathcal{Q}^{ \pm} U_{B}(\theta),
$$

the Klein factor $K_{\beta}^{ \pm}$is the transformed Klein factor of the Fermi field at zero temperature,

$$
K_{\beta}^{ \pm}=U_{B}(-\theta) K^{ \pm} U_{B}(\theta) .
$$

Starting from eqs. (5.1) and (5.2) the thermofields are redefined as,

$$
\begin{gathered}
\psi\left(x^{+} ; \beta\right)=W_{R}\left(x^{+} ; \beta\right), \\
\psi\left(x^{-} ; \beta\right)=K_{\beta}^{+} W_{R}\left(x^{-} ; \beta\right), \\
\widetilde{\psi}\left(x^{+} ; \beta\right)=K_{\beta}^{+} K_{\beta}^{-} \widetilde{W}_{R}\left(x^{+} ; \beta\right), \\
\widetilde{\psi}\left(x^{-} ; \beta\right)=K_{\beta}^{+} K_{\beta}^{-} \widetilde{K}_{\beta}^{+} \widetilde{W}_{R}\left(x^{-} ; \beta\right) .
\end{gathered}
$$

These fields obey the correct anticommutation relations. The factor $K_{\beta}^{+}$in (5.19) and $\widetilde{K}_{\beta}^{+}$in (5.21) ensures that for different (left, right ) spinor components we obtain,

$$
\left\{\psi\left(x^{ \pm} ; \beta\right), \psi\left(y^{\mp} ; \beta\right)\right\}=0
$$




$$
\left\{\widetilde{\psi}\left(x^{ \pm} ; \beta\right), \widetilde{\psi}\left(y^{\mp} ; \beta\right)\right\}=0 .
$$

The factors $K_{\beta}^{ \pm}$in (5.20) and (5.21) ensures the normal anticommutativity between tilde thermofermion components and non-tilde ones,

$$
\begin{aligned}
& \left\{\widetilde{\psi}\left(x^{ \pm} ; \beta\right), \psi\left(y^{ \pm} ; \beta\right)\right\}=0, \\
& \left\{\widetilde{\psi}\left(x^{ \pm} ; \beta\right), \psi\left(y^{\mp} ; \beta\right)\right\}=0 .
\end{aligned}
$$

\subsection{Fermionic Current and $\varepsilon$-expansion}

The vector current corresponding to the fermion doublet (4.1) is given by,

$$
\bar{\Psi} \gamma^{\mu} \Psi=\mathcal{J}^{\mu}-\widetilde{\mathcal{J}}^{\mu}
$$

where,

$$
\begin{aligned}
\mathcal{J}^{\mu} & =\bar{\psi} \gamma^{\mu} \psi, \\
\widetilde{\mathcal{J}}^{\mu} & =\overline{\widetilde{\psi}} \gamma^{\mu} \widetilde{\psi} .
\end{aligned}
$$

Let us define the finite-temperature left and right current components

$$
\begin{aligned}
& J^{ \pm}(x ; \beta)=\psi^{\dagger}\left(x^{ \pm} ; \beta\right) \psi\left(x^{ \pm} ; \beta\right), \\
& \widetilde{J}^{ \pm}(x ; \beta)=\widetilde{\psi}^{\dagger}\left(x^{ \pm} ; \beta\right) \widetilde{\psi}\left(x^{ \pm} ; \beta\right),
\end{aligned}
$$

by point-splitting as,

$$
J^{ \pm}(x ; \beta)=\lim _{\varepsilon^{ \pm} \rightarrow 0}\left\{\psi^{\dagger}\left(x^{ \pm}+\varepsilon^{ \pm} ; \beta\right) \psi\left(x^{ \pm} ; \beta\right)-\left\langle 0, \widetilde{0}\left|\psi^{\dagger}\left(x^{ \pm}+\varepsilon^{ \pm} ; \beta\right) \psi\left(x^{ \pm} ; \beta\right)\right| 0, \widetilde{0}\right\rangle\right\},
$$

and similarly for $\widetilde{J}^{ \pm}(x ; \beta)$. We obtain,

$$
\begin{gathered}
J^{ \pm}(x ; \beta)=\frac{\mu}{2 \pi} \mathcal{Z}_{c}^{2}\left(\beta, \mu^{\prime}\right) \lim _{\varepsilon^{ \pm} \rightarrow 0}\left\{: e^{-2 i \sqrt{\pi} \phi\left(x^{ \pm}+\varepsilon^{ \pm} ; \beta\right)}:: e^{2 i \sqrt{\pi} \phi\left(x^{ \pm} ; \beta\right)}:-(\text { V.E.V. })_{\beta}\right\}= \\
\frac{\mu}{2 \pi} \mathcal{Z}_{c}^{2}\left(\beta, \mu^{\prime}\right) \lim _{\varepsilon^{ \pm} \rightarrow 0}\left\{: e^{-2 i \sqrt{\pi}\left[\phi\left(x^{ \pm}+\varepsilon^{ \pm} ; \beta\right)-\phi\left(x^{ \pm} ; \beta\right)\right]}: e^{4 \pi D_{c}^{(+)}\left(\varepsilon^{ \pm} ; \beta\right)+4 \pi \widetilde{D}_{s}^{(+)}\left(\varepsilon^{ \pm} ; \beta\right)}-(V . E . V .)_{\beta}\right\}, \\
\widetilde{J}^{ \pm}(x ; \beta)=\frac{\mu}{2 \pi} \mathcal{Z}_{c}^{2}\left(\beta, \mu^{\prime}\right) \lim _{\varepsilon^{ \pm} \rightarrow 0}\left\{: e^{2 i \sqrt{\pi} \widetilde{\phi}\left(x^{ \pm}+\varepsilon^{ \pm} ; \beta\right)}:: e^{-2 i \sqrt{\pi} \widetilde{\phi}\left(x^{ \pm} ; \beta\right)}:-(V . E . V .)_{\beta}\right\}=
\end{gathered}
$$




$$
\frac{\mu}{2 \pi} \mathcal{Z}_{c}^{2}\left(\beta, \mu^{\prime}\right) \lim _{\varepsilon^{ \pm} \rightarrow 0}\left\{: e^{2 i \sqrt{\pi}\left[\widetilde{\phi}\left(x^{ \pm}+\varepsilon^{ \pm} ; \beta\right)-\widetilde{\phi}\left(x^{ \pm} ; \beta\right)\right]}: e^{4 \pi \widetilde{D}_{c}^{(+)}\left(\varepsilon^{ \pm} ; \beta\right)+4 \pi D_{s}^{(+)}\left(\varepsilon^{ \pm} ; \beta\right)}-(\text { V.E.V. })_{\beta}\right\} .
$$

Since the short-distance behavior of the thermal two-point functions (3.51) and (3.52) is the same as that for zero temperature, and the superselection rule is identically satisfied, we obtain the $\mu$ and $\mu^{\prime}$ independent result,

$$
\begin{aligned}
J\left(x^{ \pm} ; \beta\right) & =\lim _{\varepsilon^{ \pm} \rightarrow 0} \frac{1}{2 \pi i \varepsilon^{ \pm}}\left\{: e^{-2 i \sqrt{\pi} \varepsilon^{ \pm} \partial_{x^{ \pm}} \phi\left(x^{ \pm} ; \beta\right)}:-(\text { V.E.V. })_{\beta}\right\} \\
& =-\frac{1}{\sqrt{\pi}} \partial_{x^{ \pm}} \phi\left(x^{ \pm} ; \beta\right) \\
\widetilde{J}\left(x^{ \pm} ; \beta\right) & =\lim _{\varepsilon^{ \pm} \rightarrow 0} \frac{e^{-i \pi}}{2 \pi i \varepsilon^{ \pm}}\left\{: e^{2 i \sqrt{\pi} \varepsilon^{ \pm} \partial_{x^{ \pm}} \widetilde{\phi}\left(x^{ \pm} ; \beta\right)}:-(V . E . V .)_{\beta}\right\} \\
& =-\frac{1}{\sqrt{\pi}} \partial_{x^{ \pm}} \widetilde{\phi}\left(x^{ \pm} ; \beta\right)
\end{aligned}
$$

Defining the pseudoscalar thermofield $\varphi(x ; \beta)$, such that,

$$
\epsilon_{\mu \nu} \partial^{\nu} \varphi(x ; \beta)=\partial_{\mu} \phi(x ; \beta)
$$

the vector currents can be written as,

$$
\begin{aligned}
& \mathcal{J}^{\mu}(x ; \beta)=: \bar{\psi}(x ; \beta) \gamma^{\mu} \psi(x ; \beta):=-\frac{1}{\sqrt{\pi}} \epsilon^{\mu \nu} \partial_{\nu} \varphi(x ; \beta)=-\frac{1}{\sqrt{\pi}} \partial^{\mu} \phi(x ; \beta), \\
& \widetilde{\mathcal{J}}^{\mu}(x ; \beta)=: \widetilde{\bar{\psi}}(x ; \beta) \gamma^{\mu} \widetilde{\psi}(x ; \beta):=-\frac{1}{\sqrt{\pi}} \epsilon^{\mu \nu} \partial_{\nu} \widetilde{\varphi}(x ; \beta)=-\frac{1}{\sqrt{\pi}} \partial^{\mu} \widetilde{\phi}(x ; \beta) .
\end{aligned}
$$

From (5.34), the current two-point function is found to be given by,

$$
\begin{aligned}
\left\langle 0, \widetilde{0}\left|J^{ \pm}(x ; \beta) J^{ \pm}(y ; \beta)\right| 0, \widetilde{0}\right\rangle=\quad \frac{1}{\pi} & \partial_{x^{ \pm}} \partial_{y^{ \pm}}\langle 0, \widetilde{0}|\phi(x ; \beta) \phi(y ; \beta)| 0, \widetilde{0}\rangle \\
& =\frac{-1}{4 \beta^{2}} \frac{1}{\sinh ^{2}\left[\frac{\pi}{\beta}\left(x^{ \pm}-y^{ \pm}-i \epsilon\right)\right]}
\end{aligned}
$$

In the zero temperature limit we recover the zero temperature correlation function,

$$
\left\langle 0\left|J^{ \pm}(x) J^{ \pm}(y)\right| 0\right\rangle=\frac{-1}{4 \pi^{2}} \frac{1}{\left(x^{ \pm}-y^{ \pm}-i \epsilon\right)^{2}} .
$$

An alternative way to obtain the bosonized form of the current (15.34) is to consider the expectation value of the $T=0$ current with respect to the $|\beta, 0\rangle$ vacuum. To this end, let us again define the current two-point function in terms of a point-splitting, 


$$
\begin{gathered}
\left\langle 0(\beta)\left|J^{ \pm}(x) J^{ \pm}(y)\right| 0(\beta)\right\rangle= \\
\lim _{\varepsilon^{ \pm}, \delta^{ \pm} \rightarrow 0}\left\langle 0(\beta)\left|\left(\psi^{\dagger}\left(x^{ \pm}+\varepsilon^{ \pm}\right) \psi\left(x^{ \pm}\right)\right)\left(\psi^{\dagger}\left(y^{ \pm}+\delta^{ \pm}\right) \psi\left(y^{ \pm}\right)\right)\right| 0(\beta)\right\rangle= \\
\left(\frac{\mu}{2 \pi}\right)^{2} \mathcal{Z}_{c}^{4}\left(\beta, \mu^{\prime}\right) \lim _{\varepsilon^{ \pm}, \delta^{ \pm} \rightarrow 0}\left\langle 0, \widetilde{0}\left|\left(W^{*}\left(x^{ \pm}+\varepsilon^{ \pm} ; \beta\right) W\left(x^{ \pm} ; \beta\right)\right)\left(W^{*}\left(y^{ \pm}+\delta^{ \pm} ; \beta\right) W\left(y^{ \pm} ; \beta\right)\right)\right| \widetilde{0}, 0\right\rangle .
\end{gathered}
$$

Since the superselection rule is identically satisfied, the dependence on $\mu$ and $\mu^{\prime}$ cancels and we get,

$$
\begin{gathered}
\left\langle 0(\beta)\left|J^{ \pm}(x) J^{ \pm}(y)\right| 0(\beta)\right\rangle= \\
\left(\frac{\mu}{2 \pi}\right)^{2} \lim _{\varepsilon^{ \pm}, \delta^{ \pm} \rightarrow 0}\left(\frac{-1}{\mu^{2} \varepsilon \delta}\right)\left\langle 0, \widetilde{0}\left|\left(1-2 i \sqrt{\pi} \varepsilon^{ \pm} \partial_{x^{ \pm}} \phi\left(x^{ \pm} ; \beta\right)\right)\left(1-2 i \sqrt{\pi} \delta^{ \pm} \partial_{y^{ \pm}} \phi\left(y^{ \pm} ; \beta\right)\right)\right| \widetilde{0}\right. \\
\left.=\frac{1}{\pi}\langle 0, \widetilde{0}| \partial^{x^{ \pm}} \phi\left(x^{ \pm} ; \beta\right) \partial_{y^{ \pm}} \phi\left(y^{ \pm} ; \beta\right)\right)|\widetilde{0}, 0\rangle-\frac{1}{4 \pi^{2} \varepsilon^{ \pm} \delta^{ \pm}} .
\end{gathered}
$$

The singular term appearing in (5.42) can be removed by redefining the point-splitting by subtracting the contribution coming from the vacuum expectation value of the individual current operators, i. e.,

$$
\begin{gathered}
\left\langle 0(\beta)\left|J^{ \pm}(x) J^{ \pm}(y)\right| 0(\beta)\right\rangle= \\
\lim _{\varepsilon, \delta^{ \pm} \rightarrow \pm 0}\left\{\left\langle 0(\beta)\left|\left(\psi^{\dagger}\left(x^{ \pm}+\varepsilon^{ \pm}\right) \psi\left(x^{ \pm}\right)\right)\left(\psi^{\dagger}\left(y^{ \pm}+\delta^{ \pm}\right) \psi\left(y^{ \pm}\right)\right)\right| 0(\beta)\right\rangle-\right. \\
\left.-\left\langle 0(\beta)\left|\psi^{\dagger}\left(x^{ \pm}+\varepsilon^{ \pm}\right) \psi\left(x^{ \pm}\right)\right| 0(\beta)\right\rangle\left\langle 0(\beta)\left|\psi^{\dagger}\left(y^{ \pm}+\delta^{ \pm}\right) \psi\left(y^{ \pm}\right)\right| 0(\beta)\right\rangle\right\} .
\end{gathered}
$$

The same for $\widetilde{J}^{ \pm}\left(x^{ \pm} ; \beta\right)$.

\subsection{Bosonized Lagrangian}

The total Lagrangian density of the free massless Dirac thermofield,

$$
\mathcal{L}_{T}=i \bar{\Psi}(x ; \beta) \gamma^{\mu} \partial_{\mu} \Psi(x ; \beta)=\mathcal{L}-\widetilde{\mathcal{L}}=i \bar{\psi}(x ; \beta) \gamma^{\mu} \partial_{\mu} \psi(x ; \beta)-\left(-i \overline{\widetilde{\psi}}(x ; \beta) \gamma^{\mu} \partial_{\mu} \widetilde{\psi}(x ; \beta)\right)
$$

is equivalently given by,

$$
\mathcal{L}_{T}^{b o s}=\frac{1}{2}: \partial_{\mu} \phi(x ; \beta) \partial^{\mu} \phi(x ; \beta):-\frac{1}{2}: \partial_{\mu} \widetilde{\phi}(x ; \beta) \partial^{\mu} \widetilde{\phi}(x ; \beta): .
$$


The demonstration follows along the same lines as that given in Appendix E in [1] for the zero temperature case. To this end, we must consider the Wilson short distance expansion for the total Hamiltonian density

$$
\mathcal{H}_{T}=i \bar{\Psi} \gamma^{1} \partial_{1} \Psi=\mathcal{H}-\widetilde{\mathcal{H}}=i \bar{\psi} \gamma^{1} \partial_{1} \psi-\left(-i \overline{\widetilde{\psi}} \gamma^{1} \partial_{1} \widetilde{\psi}\right)+\text { h.c. . }
$$

Since the short-distance behavior of the thermofield is the same as that of zero temperature, we find

$$
\begin{aligned}
\mathcal{H}(x ; \beta) & =\frac{1}{2}\left(\bar{\psi}(x+\varepsilon ; \beta) i \gamma^{1} \partial_{1} \psi(x ; \beta)+\text { h.c. }\right)-(\text { V.E.V. })_{\beta} \\
& =\frac{1}{2}\left[:\left(\partial_{0} \phi(x ; \beta)\right)^{2}:+:\left(\partial_{1} \phi(x ; \beta)\right)^{2}:\right] .
\end{aligned}
$$

For $\widetilde{\mathcal{H}}$ we find,

$$
\begin{gathered}
\widetilde{\mathcal{H}}(x ; \beta)=-\frac{1}{2}\left(\overline{\widetilde{\psi}}(x+\varepsilon ; \beta) i \gamma^{1} \partial_{1} \widetilde{\psi}(x ; \beta)+\text { h.c. }\right)-(\text { V.E.V. })_{\beta} \\
=-e^{-i \pi} \frac{1}{2}\left[:\left(\partial_{0} \widetilde{\phi}(x ; \beta)\right)^{2}:+:\left(\partial_{1} \widetilde{\phi}(x ; \beta)\right)^{2}:\right] .
\end{gathered}
$$

The phase factor $e^{-i \pi}$ in (5.48), that cancels the global minus sign, comes from the phase (3.79) associated with the Wick exponential $\widetilde{W}(x ; \beta)$. The total Hamiltonian density $\mathcal{H}_{T}=\mathcal{H}-\widetilde{\mathcal{H}}$ corresponds to the total Lagrangian density (5.45) for the free massless scalar thermofield.

\section{Massless Thirring Model}

In this section we illustrate the use of the thermofield bosonization by solving the massless Thirring model, which is defined by the Lagrangian density

$$
\mathcal{L}_{T}=i \bar{\psi} \gamma^{\mu} \partial_{\mu} \psi+\frac{1}{2} g\left(\bar{\psi} \gamma^{\mu} \psi\right)\left(\bar{\psi} \gamma_{\mu} \psi\right)-\left(-i \tilde{\bar{\psi}} \gamma^{\mu} \partial_{\mu} \widetilde{\psi}+\frac{1}{2} g\left(\tilde{\bar{\psi}} \gamma^{\mu} \widetilde{\psi}\right)\left(\widetilde{\bar{\psi}} \gamma_{\mu} \widetilde{\psi}\right)\right)
$$

To begin with, let us consider the operator solution of the (zero temperature) massless Thirring model,

$$
\begin{gathered}
\psi(x)=\frac{1}{\sqrt{\pi}}(\mu)^{\frac{1}{4 \pi}\left(\alpha^{2}+\delta^{2}\right)}: e^{i\left(\alpha \gamma^{5} \varphi(x)+\delta \phi(x)\right)}:, \\
\widetilde{\psi}(x)=\frac{1}{\sqrt{\pi}}(\mu)^{\frac{1}{4 \pi}\left(\alpha^{2}+\delta^{2}\right)}: e^{-i\left(\alpha \gamma^{5} \widetilde{\varphi}(x)+\delta \widetilde{\phi}(x)\right)}:,
\end{gathered}
$$

where $\varphi(\widetilde{\varphi})$ is the dual of $\phi(\widetilde{\phi})$ and $\mu$ is the infrared regulator of the two-dimensional free massless scalar field. The $\mu$-dependent factor in front of (6.2) is needed in order to obtain in the IR limit $\mu \rightarrow 0$ well defined Wick exponentials living in a positive-metric Hilbert space. The corresponding thermofield is given by, 


$$
\psi(x ; \beta)=\frac{1}{\sqrt{\pi}}(\mu)^{\frac{1}{4 \pi}\left(\alpha^{2}+\delta^{2}\right)} \mathbf{Z}\left(\beta, \mu^{\prime}\right): e^{i\left(\alpha \gamma^{5} \varphi(x ; \beta)+\delta \phi(x ; \beta)\right)}:,
$$

where

$$
\mathbf{Z}\left(\beta, \mu^{\prime}\right)=e^{-\frac{\alpha^{2}+\delta^{2}}{\pi} z\left(\beta, \mu^{\prime}\right)-\alpha \delta \gamma^{5}\left(\left[\widetilde{\varphi}_{s}^{(+)}(x+\varepsilon ; \beta), \widetilde{\phi}_{s}^{(-)}(x ; \beta)\right]+\left[\widetilde{\phi}_{s}^{(+)}(x+\varepsilon ; \beta), \widetilde{\varphi}_{s}^{(-)}(x ; \beta)\right]\right),}
$$

and similarly for $\widetilde{\psi}(x ; \beta)$. We shall consider in particular

$$
\delta=\frac{\pi}{\alpha}
$$

in order to obtain in the zero temperature limit a canonical fermion field with Lorentz spin $s=\frac{1}{2}$,

$$
s=\frac{\alpha \delta}{2 \pi}=\frac{1}{2} .
$$

Taking into account that the short-distance behavior of the thermofield two-point function is the same as that of zero temperature, we use the Mandelstam prescription [13, 1] and define the current through the point-splitting,

$$
\mathcal{J}^{\mu}(x ; \beta)=\lim _{\substack{\varepsilon=\left(0, \varepsilon^{1}\right) \\ \varepsilon^{1} \rightarrow 0}} \mathcal{F}(\varepsilon)\left(\delta_{\mu}^{0}+\frac{\alpha^{2}}{\pi} \delta_{\mu}^{1}\right)\left[\bar{\psi}(x+\varepsilon ; \beta) \gamma^{\mu} \psi(x ; \beta)-\left\langle 0, \widetilde{0}\left|\bar{\psi}(x+\varepsilon ; \beta) \gamma^{\mu} \psi(x ; \beta)\right| \widetilde{0}, 0\right\rangle\right]
$$

where,

$$
\mathcal{F}(\varepsilon)=\left(-\mu^{2} \varepsilon^{2}\right)^{\frac{\alpha^{2}+\delta^{2}-2 \pi}{4 \pi}} .
$$

One finds,

$$
\begin{aligned}
& \mathcal{J}^{\mu}(x ; \beta)=-\frac{\alpha}{2 \pi} \epsilon^{\mu \nu} \partial_{\nu} \varphi(x ; \beta), \\
& \widetilde{\mathcal{J}}^{\mu}(x ; \beta)=-\frac{\alpha}{2 \pi} \epsilon^{\mu \nu} \partial_{\nu} \widetilde{\varphi}(x ; \beta) .
\end{aligned}
$$

The quantum equations of motion are given in terms of the symmetrized form of the interaction term of the Thirring current with the Fermi thermofields by,

$$
\begin{gathered}
i \gamma^{\mu} \partial_{\mu} \psi(x ; \beta)=-g\left\{\mathcal{J}_{\mu}(x ; \beta) \gamma^{\mu}, \psi(x ; \beta)\right\}, \\
i \gamma^{\mu} \partial_{\mu} \widetilde{\psi}(x ; \beta)=g\left\{\widetilde{\mathcal{J}}_{\mu}(x ; \beta) \gamma^{\mu}, \widetilde{\psi}(x ; \beta)\right\},
\end{gathered}
$$

with the following identification

$$
\alpha^{2}=\frac{4 \pi}{1-\frac{g}{\pi}} .
$$

The unmixed correlation functions for the thermofield spinor components $\psi_{\alpha}(x ; \beta)$ are given by, 


$$
\begin{gathered}
\left.\left\langle 0, \widetilde{0}\left|\psi_{\alpha}\left(x_{1} ; \beta\right) \cdots \psi_{\alpha}\left(x_{n} ; \beta\right) \psi_{\alpha}^{\dagger}\left(y_{1} ; \beta\right) \cdots \psi_{\alpha}^{\dagger}\left(y_{n} ; \beta\right)\right| \widetilde{0}, 0\right\rangle=(2 \pi)^{-n}(i)\right]^{-\frac{n}{2 \pi}\left(\delta^{2}+\frac{\pi}{\delta^{2}}\right)} \times \\
\prod_{i<j}^{n}\left[\frac{\beta}{\pi} \sinh \frac{\pi}{\beta}\left(x_{i}^{+}-x_{j}^{+}-i \epsilon\right)\right]^{\frac{1}{4 \pi}\left(\delta^{2}+\frac{\pi^{2}}{\delta^{2}}+2 \pi \gamma_{\alpha \alpha}^{5}\right)} \prod_{i, j}^{n}\left[\frac{\beta}{\pi} \sinh \frac{\pi}{\beta}\left(x_{i}^{-}-x_{j}^{-}-i \epsilon\right)\right]^{\frac{1}{4 \pi}\left(\delta^{2}+\frac{\pi^{2}}{\delta^{2}}-2 \pi \gamma_{\alpha \alpha}^{5}\right)} \times \\
\prod_{i<j}^{n}\left[\frac{\beta}{\pi} \sinh \frac{\pi}{\beta}\left(y_{i}^{+}-y_{j}^{+}-i \epsilon\right)\right]^{\frac{1}{4 \pi}\left(\delta^{2}+\frac{\pi^{2}}{\delta^{2}}+2 \pi \gamma_{\alpha \alpha}^{5}\right)} \prod_{i, j}^{n}\left[\frac{\beta}{\pi} \sinh \frac{\pi}{\beta}\left(y_{i}^{-}-y_{j}^{-}-i \epsilon\right)\right]^{\frac{1}{4 \pi}\left(\delta^{2}+\frac{\pi^{2}}{\delta^{2}}-2 \pi \gamma_{\alpha \alpha}^{5}\right)} \times \\
\prod_{i, j}^{n}\left[\frac{\beta}{\pi} \sinh \frac{\pi}{\beta}\left(x_{i}^{+}-y_{j}^{+}-i \epsilon\right)\right]^{-\frac{1}{4 \pi}\left(\delta^{2}+\frac{\pi^{2}}{\delta^{2}}+2 \pi \gamma_{\alpha \alpha}^{5}\right)} \prod_{i, j}^{n}\left[\frac{\beta}{\pi} \sinh \frac{\pi}{\beta}\left(x_{i}^{-}-y_{j}^{-}-i \epsilon\right)\right]^{-\frac{1}{4 \pi}\left(\delta^{2}+\frac{\pi^{2}}{\delta^{2}}-2 \pi \gamma_{\alpha \alpha}^{5}\right)}
\end{gathered}
$$

which represents the generalization of the $T=0$ result to $T \neq 0$.

\section{Conclusion}

Using the framework of Thermofield Dynamics [6] we have shown how the familiar $T=0$ bosonization formulae for fermions in $1+1$ dimensions generalize in a natural way to the case of non-zero temperature. Despite the appearance of now two infrared regulating parameters it was shown that the superselection rule already familiar from the $T=0$ case guaranteed the independence of physical quantities of both these parameters. The "Mandelstam representation" for thermal fermions now allows us to obtain in a compact way the exact and complete solution of a number of integrable models involving massless fermions in $1+1$ dimensions.

The next step would now address the problem of massive fermions in $1+1$ dimensions. Do we again have an equivalence between the Massive Thirring model and the sine-Gordon theory ? We expect that this is indeed the case, since such an equivalence has been demonstrated for correlators of fermion and sine-Gordon thermal fields in the imaginary time formalism [2]. Taking a Mandelstam representation in terms of a thermal sine-Gordon field as a starting point, can we prove, in a way analogous to what was done in the $T=0$ massive Schwinger model [12, that the bosonized thermal Dirac field satisfies the free massive Dirac equation? These and other questions will be subject of future investigations.

\section{Acknowledgments}

We are grateful to the CNPq-DAAD scientific exchange program which makes this collaboration possible.

\section{Appendix}

The purpose of this appendix is to show that the theory of the free massless scalar thermofield can be constructed as the zero mass limit of the free massive scalar thermofield theory. To begin with, let us consider the free massive scalar thermofield,

$$
\Sigma(x ; \beta)=U\left[\theta_{B}(\beta)\right] \Sigma(x) U\left[\theta_{B}(\beta)\right]=
$$




$$
\begin{aligned}
\frac{1}{2 \sqrt{\pi}} \int_{-\infty}^{+\infty} & \frac{d p}{\sqrt{p^{2}+m^{2}}}\left\{f_{p}(x)\left(a(p) \cosh \theta_{B}(p ; \beta)-\widetilde{a}^{\dagger}(p) \sinh \theta_{B}(p ; \beta)\right)\right. \\
& \left.+f_{p}^{*}(x)\left(a^{\dagger}(p) \cosh \theta_{B}(p ; \beta)-\widetilde{a}(p) \sinh \theta_{B}(p ; \beta)\right)\right\} .
\end{aligned}
$$

The thermal two-point function is given by,

$$
\langle 0, \widetilde{0}|\Sigma(x ; \beta) \Sigma(y ; \beta)| \widetilde{0}, 0\rangle=D_{o}^{(+)}(x-y ; m)+\mathcal{I}(x-y ; \beta, m),
$$

where $D_{o}^{(+)}(x-y ; m)$ is the corresponding zero temperature two-point function,

$$
D_{o}^{(+)}(x-y ; m)=\frac{1}{4 \pi} \int_{0}^{\infty} \frac{d p}{\sqrt{p^{2}+m^{2}}}\left\{e^{-i\left[\left(x^{0}-y^{0}\right) \sqrt{p^{2}+m^{2}}+\left(x^{1}-y^{1}\right) p\right]}+e^{-i\left[\left(x^{0}-y^{0}\right) \sqrt{p^{2}+m^{2}}-\left(x^{1}-y^{1}\right) p\right]}\right\}
$$

and the last integral in (A.2) can be written as,

$$
\begin{gathered}
\mathcal{I}(x-y ; \beta, m)=\frac{1}{2 \pi} \int_{0}^{\infty} \frac{d p}{\sqrt{p^{2}+m^{2}}} N_{B}(w, \beta)\left\{\cos \left[\left(x^{0}-y^{0}\right) \sqrt{p^{2}+m^{2}}+\left(x^{1}-y^{1}\right) p\right]\right. \\
\left.+\cos \left[\left(x^{0}-y^{0}\right) \sqrt{p^{2}+m^{2}}-\left(x^{1}-y^{1}\right) p\right]\right\}
\end{gathered}
$$

where

$$
N_{B}(w, \beta)=\frac{1}{e^{\beta w}-1}=\frac{1}{e^{\beta \sqrt{p^{2}+m^{2}}}-1} .
$$

As long as $m \neq 0$ the integrals (A.3) and (A.4) are not infrared divergent. Thus, before considering the zero mass limit an infrared cut-off $\mu>0$ must be introduced. In the limit $m \rightarrow 0, N_{B}(w, \beta) \rightarrow$ $N_{B}(p ; \beta)$ and we obtain,

$$
D^{(+)}(x-y ; m, \beta)_{m \rightarrow 0}=D_{o}^{(+)}(x-y)+\frac{1}{2 \pi} \int_{\mu}^{\infty} \frac{d p}{p} N_{B}(p ; \beta)\left[\cos p\left(x^{+}-y^{+}\right)+\cos p\left(x^{-}-y^{-}\right)\right],
$$

which is the two-point function of the free massless thermofield given by Eq. (3.44). By considering the free massless scalar thermofield theory as the zero mass limit of the free massive scalar thermofield theory, the infrared regulator $\mu$ of the zero temperature two-point function should be identified with the infrared cut-off $\mu^{\prime}$ of the temperature dependent contribution in (3.44). 


\section{References}

[1] E. Abdalla, M.C.B. Abdalla and K.D. Rothe, "Non-Perturbative Methods in two dimensional Quantum Field Theory", World Scientific, Singapore, 1991, and idem. 2nd edition, 2001.

[2] D. Delepine, R. Gonzales Felipe and J. Weyers, Phys. Lett. 419 (1998) 296 ; A. Gomez Nicola and D.A. Steer, Nucl. Phys. B549 (1998) 409; A. Gomez Nicola, R.J. Rivers and D.A. Steer, Nucl. Phys. B570 (2000) 475.

[3] H. Umezawa, H. Matsumoto, M. Tachiki, "Thermo Field Dynamics and Condensed States", Nort-Holland 1982;

[4] I. Ojima, Ann. phys. 137 (1981) 1;

[5] H. Matsumoto, I. Ojima and H. Umezawa, Ann. Phys. 152 (1984) 348.

[6] N.P. Landsman and Ch.G. van Weert, Physics Reports 145 (1987) 141; A. Das, "Finite Temperature Field Theory", World Scientific, 1997.

[7] N.K. Nielsen, K.D. Rothe and B. Schroer, Nucl. Phys. B160 (1979) 330.

[8] L. Dolan and R. Jackiw, Phys. Rev. D9 (1974) 3320.

[9] J. Gamboa, J. Lopez-Sarrion, M. Loewe and F. Mendez, Mod. Phys. Lett. A19 (2004) 223.

[10] O. Klein, J. Phys. Radium 9 (1938) 1; G. Lüders, Z. Naturforsch. A 13 (1958) 254; H. Araki, J. Math. Phys. 2 (1961) 267;

[11] R. Jost , "The General Theory of Quantized Fields ", Amer. Math. Soc., Providence, R. I. 1965; R. F. Streater and A. S. Wightman, "PCT, Spin and Statistics, and all that", Benjamim, New York, 1964;

[12] K.D. Rothe and J.A. Swieca, Phys. Rev. D15 (1977) 1675.

[13] S. Mandelstam, Phys. Rev. D11 (1975)3026. 